\title{
Improvability Theory for Assembly Systems: Two Component-One Assembly Machine Case
}

\author{
C.-T. KUO ${ }^{a}$, J.-T. LIM ${ }^{b}$, S. M. MEERKOV ${ }^{a}$ and E. PARK ${ }^{\mathrm{C}}$ \\ a Department of Electrical Engineering and Computer Science, University \\ of Michigan, Ann Arbor, MI 48109-2122; ' On Sabbatical leave from the \\ Department of Electrical Engineering, Korea Advanced Institute of \\ Science and Technology, Taejon, Korea; ' ${ }^{\circ}$ Department of Aerospace \\ Engineering, University of Michigan, Ann Arbor, MI 48109-2140
}

(Received 18 April 1996)

Improvability theory for a simple assembly system consisting of two components and one assembly machine is developed. Both constrained and unconstrained formulations are addressed. In the constrained case, it is shown that the assembly is unimprovable with respect to workforce if each component machine is blocked as frequently as the assembly machine is starved for parts produced by this particular assembly machine. The system is unimprovable with respect to work-in-process if, roughly speaking, all buffers have equal average steady state occupancy. In the unconstrained improvability case, it is shown that the bottleneck machine can be identified by analyzing the probabilities of the so-called manufacturing blockages and starvations. A generalization to $n$ component—one assembly machine system is also included.

Keywords: Improvability; assembly systems; bottlenecks; manufacturing blockage and starvation

\section{INTRODUCTION}

Assembly is a production system which includes the merging of parts produced. In most cases, it consists of two or more component machines, one or more assembly machines and, if necessary, additional processing machines. All the machines are separated by finite buffers. An example of 
an assembly is shown in Figure 1, where $m_{i j}, i>0$, are the component machines, $m_{01}$ is the assembly machine, $m_{0 j}, j>1$, are the additional processing machines, and $b_{i j}$ and $b_{0 j}$ are the buffers. Systems of this structure are the focus of this paper.

The idea of improvability for production systems was introduced in [1] to facilitate the development of quantitative engineering methods for design and implementation of continuous improvement projects on the factory floor. Roughly speaking, a production system is called improvable under constraints if limited resources involved in its operation (for instance, workforce-WF or work-in-process-WIP) can be re-distributed so that a performance index is improved. Unconstrained improvability addresses the question of constraints relaxation and, in particular, the identification of the machine improvement of which results in the best possible behavior of the system as a whole.

The properties of improvability under constraints for the simplest class of production systems-serial lines, where all machines are arranged in the consecutive order-have been investigated in [1], [2]. In particular, it has been shown that a serial line is unimprovable with respect to the WF if and only if all of its buffers are on the average half full. In other words, if some of the system's buffers are too full, the system can be improved; analogously, when some of the buffers are too empty; only when all the buffers are on the average half full (whatever the size of buffers may to be), the system is unimprovable by re-assigning the existing WF. Also, it has been shown that a serial line is unimprovable with respect to WIP and WF simultaneously if and only if each machine is blocked and starved with equal probability. In a certain sense (see [2]), this condition holds

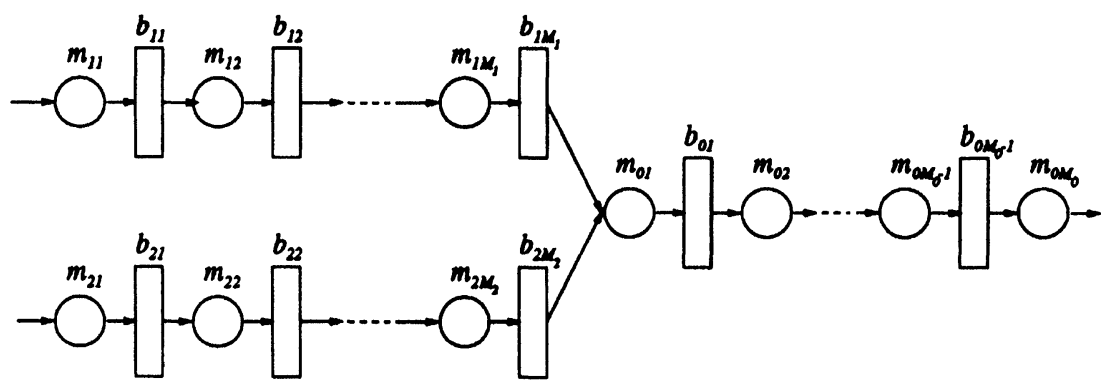

FIGURE 1 Assembly system. 
also for improvability with respect to WIP. Thus, if some of the machines in the system are blocked more often than they are starved, the system is improvable; analogously, if some of the machines are more often starved than blocked; only when each machine is blocked and starved with equal frequency, the system is unimprovable by WIP re-allocation. Several extensions of this theory and a practical application have been reported in [3]-[5].

The unconstrained improvability for serial lines was analyzed in [2] and [6]. The bottleneck was defined as the machine which has the largest effect onto the overall system production rate. It was shown that in a system unimprovable with respect to WF, the slowest machine is always the bottleneck. Systems which are improvable with respect to WF may or may not have the slowest machine as the bottleneck. Sometimes other machines, including the fastest one, could be the bottleneck. In [6], a method for bottlenecks identification was developed, and it was shown that they can be identified by analyzing the probabilities of the so-called manufacturing blockages and starvations.

Although assembly systems have been a subject of many studies over the years (see [7], [8] and references therein) and heuristic algorithms for their performance analysis have been (relatively recently) developed ([9][11]), system-theoretic properties of these systems remain largely unknown. Two exceptions are reported in [12], [13], where the question of work and work-in-process distribution among the component machines and the assembly machine has been addressed. In particular, it has been shown that less work should be assigned to the assembly machine than to each of the component machines.

In this paper, we develop improvability theory for a simple assembly system consisting of two component and one assembly machine. Both constrained and unconstrained improvability are addressed. A generalization for $n$ component machines, working in parallel, is also presented. The case of a more general systems, such as shown in Figure 1, will be described elsewhere.

The goal of the development presented here is not only to characterize the improvability properties of the assembly but also to accomplish this in terms of variables available on the factory floor. This should make the results obtained practical from the point of view of applications. Indeed, conditions formulated in terms which are either unfamiliar to practitioners or are not measurable on the factory floor could hardly find a receptive 
audience among the production systems personnel. Only the conditions which are, on one hand, intuitively understandable and, on the other hand, use data available on the factory floor could realistically be expected to make an impact on the manufacturing practice. To this end, every condition derived in this work is re-formulated as an Improvability Indicator, i.e., a rule which, being based on real-time data, allows the production system personnel to identify whether the systems is improvable and to design steps for accomplishing this improvement.

The outline of the paper is as follows: In Section 2, the model of the system considered and the problem analyzed are formulated. In Section 3 an auxiliary result pertaining to the production rate calculation is described. Sections 4-6 and 7-8 are devoted to constrained and unconstrained improvability, respectively. A generalization for a system with $n$ component machines is outlined in Section 9. The conclusions are given in Section 10. All the proofs are assembled in Appendices A-E.

Roughly speaking, the main results of this work are as follows:

(a). The assembly system is unimprovable with respect to WF if the probability of blockage of every component machine equals to the probability of starvation of the assembly machine caused by the lack of the parts produced by this component machine.

(b). The assembly system is unimprovable with respect to WIP if the average buffer occupancy of all buffers is as close to each other as possible.

(c). In an improvable system, if the probabilities of manufacturing blockage of the component machines are larger than the probability of manufacturing starvation of the assembly machine, the latter is the bottleneck; otherwise, one of the component machines is the bottleneck, and the rule for its identification is provided.

\section{PROBLEM FORMULATION}

\subsection{The Model}

The following model is considered throughout this work:

(i) The system consists of component machines, $m_{i}, i=1,2$, an assem- 
bly machine, $m_{0}$, and buffers, $b_{i}, i=1,2$, storing the parts produced by $m_{i}, i=1,2$, respectively; one part from each buffer is required for the assembly.

(ii) All the machines have an identical cycle time, $T$; the time axis is slotted with the slot duration $T$.

(iii) Each machine is characterized by the probability, $p_{i}, i=0,1,2$, to produce a part during a time slot; these probabilities are referred to as machines' production rate in isolation.

(iv) Each buffer is characterized by its capacity, $N_{i}, i=1,2$; the buffer capacity is assumed to be finite.

(v) A component machines $m_{i}, i=1,2$, is never starved; it is blocked during a time slot if buffer $b_{i}$ is full and the assembly machine, $m_{0}$, fails to take a part from the buffer at the beginning of this time slot.

(vi) The assembly machine is never blocked; it is starved for parts, if at least one buffer $b_{i}, i=1,2$, is empty at the beginning of this time slot.

Remark 2.1: The generalization of this model to the case of $n>2$ component machines (Section 9) is obvious: in all of the above assumptions use $i=1,2, \ldots, n$, instead of $i=1,2$.

Remark 2.2: The assumption that only one part from each buffer is required for the assembly can be removed by an appropriate modification in the model. Basically, it does not restrict the generality.

Remark 2.3: On the other hand, the assumed Bernoulli model of machines reliability (assumption (iii)) is quite restrictive. In many practical situations, machines obey the Markovian, rather than the Bernoulli, reliability model, where the machines are characterized by exponentially distributed up- and down-time. As in [1]-[5], we use the Bernoulli model because, on one hand, there are assembly operations with machine' uptime process similar to a sequence independent trials and, on the other hand, this simpler model permits a more complete analytical investigation. We believe (and have a numerical evidence) that the results reported here hold qualitatively for the Markovian model as well. However, the extension of this theory to Markovian machines is the topic of the future work.

Remark 2.4: The synchronous operation, implied by (ii), is also assumed to simplify the analysis. Although somewhat restrictive, it is not unrealistic 
for operations with automated material handling system which defines the assembly tact.

Remark 2.5: The assumption of a deterministic and fixed processing time (or cycle time) of the machines (see (ii)) does reflect the situation in most large volume production industries, with automated material handling or not. The often cited in the literature random processing time is not, in our opinion, appropriate in the context of such production systems.

\subsection{Constrained Improvability}

Assume that $N_{i}$ 's and $p_{i}$ 's are constrained as follows

$$
\begin{gathered}
N_{1}+N_{2}=N^{*}, \\
p_{1} p_{2} p_{0}=p^{*} .
\end{gathered}
$$

Obviously, (2.1) can be interpreted as WIP constraint: the total inventory in the system cannot exceed $N^{*}$. As in [1], [2], equality (2.2) is interpreted as WF (or machine efficiency) constraint: the total work that can be carried out in the system is bounded by $p^{*}$, and a reassignment of the workforce (or work) among the operations leads to changes in $p_{i}$ 's compatible with (2.2).

Definition 2.1: The assembly system (i)-(vi) is improvable with respect to WF if there exists a sequence $p_{0}^{*}, p_{1}^{*}, p_{2}^{*}$ such that $p_{1}^{*} p_{2}^{*} p_{0}^{*}=p^{*}$ and

$$
\widetilde{P R}\left(p_{1}^{*}, p_{2}^{*}, p_{0}^{*}, N_{1}, N_{2}\right)>\widetilde{P R}\left(p_{1}, p_{2}, p_{0}, N_{1}, N_{2}\right)
$$

where $\widetilde{P R}$ denotes the production rate of the system, i.e., the average steady state number of parts produced by the assembly machine per cycle time.

Definition 2.2: The assembly system (i)-(vi) is improvable with respect to WF and WIP simultaneously if there exist sequences $p_{0}^{*}, p_{1}^{*}, p_{2}^{*}$ and $N_{1}^{*}, N_{2}^{*}$ such that $p_{1}^{*} p_{2}^{*} p_{0}^{*}=p^{*}, N_{1}^{*}+N_{2}^{*}=N^{*}$ and 


$$
\widetilde{P R}\left(p_{1}^{*}, p_{2}^{*}, p_{0}^{*}, N_{1}^{*}, N_{2}^{*}\right)>\widetilde{P R}\left(p_{1}, p_{2}, p_{0}, N_{1}, N_{2}\right) .
$$

Definition 2.3: The assembly system (i)-(vi) is improvable with respect to WIP if there exist $N_{1}^{*}$ and $N_{2}^{*}$ such that $N_{1}^{*}+N_{2}^{*}=N^{*}$ and

$$
\widetilde{P R}\left(p_{1}, p_{2}, p_{0}, N_{1}^{*}, N_{2}^{*}\right)>\widetilde{P R}\left(p_{1}, p_{2}, p_{0}, N_{1}, N_{2}\right)
$$

\subsection{Unconstrained Improvability}

When the system is no longer improvable under constraints or when the resource re-allocation, required by the improvability conditions, cannot be carried on the factory floor, a further increase in $\widetilde{P R}$ can be obtained only by the constraints relaxation, i.e., by increasing $p^{*}$ or $N^{*}$. In practical terms, this amounts to the improvement of the machines isolation production rates (say, by improved preventative maintenance or by installing a more efficient machine) or by allocating additional in-process inventories to the system. The question arises: Which $p_{i}$ or $N_{i}$ should be increased so that the largest increase in $\widetilde{P R}$ is obtained? A formalization of this question is as follows:

Definition 2.4: Machine $m_{i}, i=0,1,2$, is the bottleneck machine if

$$
\frac{\partial \widetilde{P R}}{\partial p_{i}}>\frac{\partial \widetilde{P R}}{\partial p_{j}}, \forall j \neq i
$$

Definition 2.5: Buffer $b_{1}$ is the bottleneck buffer if

$$
\widetilde{P R}\left(p_{1}, p_{2}, p_{0}, N_{1}+1, N_{2}\right)>\widetilde{P R}\left(p_{1}, p_{2}, p_{0}, N_{1}, N_{2}+1\right)
$$

Buffer $b_{2}$ as the bottleneck is defined analogously.

Remark 2.6: In Definitions 2.1-2.5 and throughout this paper, the symbols with the " " denote the exact values of the appropriate quantity. The respective analytical approximation, to be introduced below, is denoted by the same symbol but without the " ".

Remark 2.7: Definitions 2.1-2.5 have obvious generalizations to the $n$ component machines case: The sequences of three $p_{i}$ 's and two $N_{i}$ 's 
should be replaced by sequences of $p_{i}$ 's and $N_{j}$ 's, with $i=1, \ldots, n+1$; $j=1, \ldots, n$.

\subsection{Problems}

The goal of this paper is to derive conditions under which the assembly system (i)-(vi) is improvable in both constrained and unconstrained sense. As it was pointed out above, the conditions sought are to be formulated either in terms of the data available on the factory floor or in terms of the data that can be constructively calculated using the machines and buffers parameters, $p_{i}$ 's and $N_{i}$ 's. As in [1], [2], we refer to these conditions as Indicators of Improvability. The problems, then, addressed in this paper are:

Problem 1: Given model (i)-(vi), derive indicators of improvability with respect to WF, WIP, and WF and WIP simultaneously which could be used based on either measured or calculated data.

Problem 2: Given model (i)-(vi), derive indicators of improvability for bottleneck identification which are based on real-time or calculated data.

Solutions of Problems 1 and 2 are given in Sections 4-6 and 7-8, respectively. In the unconstrained case, only the notion of bottleneck machines is analyzed; the problem of bottleneck buffers will be addressed elsewhere.

\section{PRODUCTION RATE EVALUATION}

This section presents auxiliary results used below to provide an analytical solution to Problems 1 and 2.

Under assumption (i)-(vi), the dynamics of the assembly system are described by an irreducible, ergodic Markov chain with the states $\left(k_{1}, k_{2}\right)$, $k_{i}=1, \ldots, N_{i}, i=1,2$. Let $\tilde{X}_{1,2}\left(k_{1}, k_{2}\right)$ be the stationary probability that buffers $b_{1}$ and $b_{2}$ have $k_{1}$ and $k_{2}$ parts, respectively. In terms of $\tilde{X}_{1,2}\left(k_{1}, k_{2}\right)$, the production rate of the system is: 


$$
\begin{aligned}
\widetilde{P R} & =p_{0} \sum_{k_{2}=1}^{N_{2}} \sum_{k_{1}=1}^{N_{1}} \tilde{X}_{1,2}\left(k_{1}, k_{2}\right) \\
& =p_{0}\left[1-\tilde{X}_{1,2}(0,0)-\sum_{k_{1}=1}^{N_{1}} \tilde{X}_{1,2}\left(k_{1}, 0\right)-\sum_{k_{2}=1}^{N_{2}} \tilde{X}_{1,2}\left(0, k_{2}\right)\right] .
\end{aligned}
$$

Unfortunately, $\tilde{X}_{1,2}\left(k_{1}, k_{2}\right)$ cannot be calculated in the closed form. Therefore, a simplification is necessary. One possibility is to reduce the joint probability distribution to the product of its marginals. In general, of course, the joint probability distribution $\tilde{X}_{1,2}\left(k_{1}, k_{2}\right)$ is not equal to the product of its marginals, $\tilde{X}_{1}\left(k_{1}\right)$ and $\tilde{X}_{2}\left(k_{2}\right)$. However, when either $k_{1}$ or $k_{2}$ or both are 0 , these two quantities are close to each other in the following sense: Introduce

$$
\begin{aligned}
\mu_{1}\left(k_{1}\right) & :=\left|\tilde{X}_{1,2}\left(k_{1}, 0\right)-\tilde{X}_{1}\left(k_{1}\right) \tilde{X}_{2}(0)\right|, k_{1}=0, \ldots, N_{1}, \\
\mu_{2}\left(k_{2}\right) & :=\left|\tilde{X}_{1,2}\left(0, k_{2}\right)-\tilde{X}_{1}(0) \tilde{X}_{2}\left(k_{2}\right)\right|, k_{2}=0, \ldots, N_{2}, \\
\mu & :=\max _{k_{1}, k_{2}}\left\{\mu_{1}\left(k_{1}\right), \mu_{2}\left(k_{2}\right)\right\} .
\end{aligned}
$$

Numerical FACT 3.1: For the assembly system defined by assumptions (i)-(vi),

$$
\mu<<1 \text {. }
$$

Justification: Justification is carried out using numerical analysis of the Markov chain defined by assumptions (i)-(vi). Specifically, the transition matrix has been constructed and iterated upon until the stationary probability distribution, $\tilde{X}_{1,2}\left(k_{1}, k_{2}\right)$, has been reached. The $\tilde{X}_{1,2}\left(k_{1}, 0\right)$ and $\tilde{X}_{1,2}\left(0, k_{2}\right)$, thus calculated, have been compared with the products of their marginals $\tilde{X}_{1}\left(k_{1}\right) \tilde{X}_{2}(0)$ and $\tilde{X}_{1}(0) \tilde{X}_{2}\left(k_{2}\right)$, where

$$
\tilde{X}_{1}\left(k_{1}\right)=\sum_{k_{2}=0}^{N_{2}} \tilde{X}_{1,2}\left(k_{1}, k_{2}\right)
$$




$$
\tilde{X}_{2}\left(k_{2}\right)=\sum_{k_{1}=0}^{N_{1}} \tilde{X}_{1,2}\left(k_{1}, k_{2}\right) .
$$

Finally, the value of $\mu$ has been calculated. In every case analyzed, it was found that $\mu<1$. Several typical example are shown in Table I. Based on this evidence, we conclude that Numerical Fact 3.1 holds.

Remark 3.1: We believe that Numerical Fact 3.1 can be proved analytically. Up to now, however, we were unable to do so. The readers are certainly encouraged to attempt such a proof.

Using Numerical Fact 3.1, expression (3.1) can be rewritten as follows:

$$
\begin{gathered}
\widetilde{P R}=p_{0}\left[1-\tilde{X}_{1}(0) \tilde{X}_{2}(0)-\sum_{k_{1}=1}^{N_{1}} \tilde{X}_{1}\left(k_{1}\right) \tilde{X}_{2}(0)\right. \\
\left.-\sum_{k_{2}=1}^{N_{2}} \tilde{X}_{1}(0) \tilde{X}_{2}\left(k_{2}\right)\right]+O(\mu) \\
=p_{0}\left[1-\tilde{X}_{1}(0) \tilde{X}_{2}(0)-\left(1-\tilde{X}_{1}(0)\right) \tilde{X}_{2}(0)\right. \\
\left.-\tilde{X}_{1}(0)\left(1-\tilde{X}_{2}(0)\right)\right]+\mathcal{O}(\mu) .
\end{gathered}
$$

Therefore,

$$
\widetilde{P R}=p_{0}\left[1-\tilde{X}_{1}(0)\right]\left[1-\tilde{X}_{2}(0)\right]+\mathcal{O}(\mu)
$$

The quantities $\tilde{X}_{1}(0)$ and $\tilde{X}_{2}(0)$, i.e., the probabilities that buffers $b_{1}$ and $b_{2}$ are empty, still cannot be calculated in the closed form. However, a recursive evaluation is possible. Indeed, assume that the estimate of the

TABLE I Illustration of $\mu$ and the estimation error

\begin{tabular}{ccccccccc}
\hline$p_{1}$ & $p_{2}$ & $p_{0}$ & $N_{1}$ & $N_{2}$ & $\mu$ & $\widetilde{P R}$ & $P R$ & $\frac{\widetilde{P R}-P R \mid}{\widetilde{P R}} \cdot 100 \%$ \\
\hline 0.7 & 0.6 & 0.8 & 1 & 1 & 0.0032 & 0.4527 & 0.4512 & $0.3 \%$ \\
0.8 & 0.7 & 0.8 & 1 & 2 & 0.0024 & 0.598 & 0.5974 & $0.1 \%$ \\
0.7 & 0.6 & 0.8 & 2 & 2 & 0.0108 & 0.5492 & 0.5537 & $0.8 \%$ \\
0.6 & 0.6 & 0.99 & 3 & 3 & 0.029 & 0.5584 & 0.5856 & $4.9 \%$ \\
0.9 & 0.9 & 0.6 & 2 & 2 & 0.0013 & 0.5893 & 0.589 & $0.05 \%$ \\
\hline
\end{tabular}


probability that $b_{2}$ is empty at the $s$-th step of the recursion is known; denote this probability as $X_{2}(0, s)$. Consider the serial line $\left\{m_{1}, b_{1}, m_{0}\right\}$ (see Figure 2) with the isolation production rate of machines $m_{1}$ and $m_{0}$ defined as $p_{1}$ and $p_{0}\left[1-X_{2}(0, s)\right]$, respectively. For this line, using the results of [1], it is easy to calculate the probability that $b_{1}$ is empty. Specifically, this probability is $Q\left(p_{1}, p_{0}\left[1-X_{2}(0, s)\right], N_{1}\right)$, where function $Q(x, y, N)$ is defined below and $N_{1}$ is the capacity of $b_{1}$. Denote this probability as $X_{1}(0, s+1)$ and consider another serial line, $\left\{m_{2}, b_{2}, m_{0}\right\}$, with the isolation production rate of $m_{2}$ and $m_{0}$ given by $p_{2}$ and $p_{0}[1-$ $X_{1}(0, s+1)$ ], respectively. Again, calculate the probability that $b_{2}$ is empty and, thus, obtain $X_{2}(0, s+1)$. As a result, we obtain the following recursive procedure:

$$
\begin{aligned}
X_{1}(0, s+1) & =Q\left(p_{1}, p_{0}\left[1-X_{2}(0, s)\right], N_{1}\right), \\
X_{2}(0, s+1) & =Q\left(p_{2}, p_{0}\left[1-X_{1}(0, s+1)\right], N_{2}\right), \\
X_{2}(0,0) & =0, s=0,1,2, \ldots,
\end{aligned}
$$

where

$$
Q(x, y, N)\left\{\begin{array}{cc}
\frac{(1-x)(1-\alpha)}{1-\frac{x}{y} \alpha^{N}}, & x \neq y, \\
=\frac{1-x}{N+1-x}, & x=y,
\end{array} \quad \alpha=\frac{x(1-y)}{y(1-x)} .\right.
$$

Lemma 3.1: Recursive procedure (3.3) is convergent, i.e., the following limits exist:

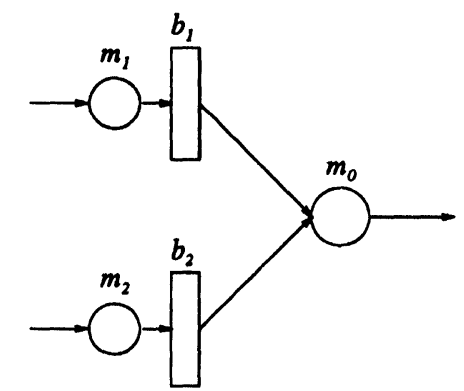

FIGURE 2 Two component-one assembly machine system. 


$$
\begin{aligned}
& \lim _{s \rightarrow \infty} X_{1}(0, s)=: X_{1}(0), \\
& \lim _{s \rightarrow \infty} X_{2}(0, s)=: X_{2}(0) .
\end{aligned}
$$

Moreover, sequences $X_{1}(0, s)$ and $X_{2}(0, s)$ are monotonically decreasing and increasing, respectively.

\section{Proof: See Appendix A.}

The relationship between the exact, $\tilde{X}_{i}(0)$, and the calculated, $X_{i}(0)$, probabilities of the buffers being empty is given by

THEOREM 3.1: For assembly lines defined by assumptions (i)-(vi),

$$
\begin{aligned}
& \tilde{X}_{1}(0)=X_{1}(0)+\mathscr{O}(\mu), \\
& \tilde{X}_{2}(0)=X_{2}(0)+\mathscr{O}(\mu) .
\end{aligned}
$$

\section{Proof: See Appendix A.}

Introduce the production rate estimate as follows:

$$
P R:=p_{0}\left[1-X_{1}(0)\right]\left[1-X_{2}(0)\right]
$$

where $X_{1}(0)$ and $X_{2}(0)$ are the steady states of (3.3).

COROllary 3.1: For the assembly system defined by assumptions (i)-(vi),

$$
\widetilde{P R}=P R+O(\mu)
$$

Proof: Follows immediately from (3.2), (3.4) and (3.5).

The numerical accuracy of the calculated production rate, defined by (3.5), is illustrated in Table I, where both $\widetilde{P R}$ and $P R$ are shown along with the approximation error. All cases analyzed resulted in a high accuracy, with $0.05 \%-5 \%$ error. Based upon this evidence, the improvability analysis described in this paper is carried out in terms of the calculated production rate, $P R$, defined by (3.3), (3.5).

To conclude this Section, we cite two more auxiliary results. The first one is concerned with the relationship between the exact and the calcu- 
lated steady state occupancies of buffers $b_{1}$ and $b_{2}$ and the second describes the monotonicity properties of the assembly system.

Let

$\tilde{X}_{i}\left(k_{i}\right)=\operatorname{Prob}\left\{b_{i}\right.$ has $k_{i}$ parts in the steady state $\}, k_{i}=1, \ldots, N_{i}, i=1,2$.

Introduce

$$
\begin{aligned}
& X_{1}\left(k_{1}\right)=X_{1}(0)\left(\frac{1}{1-p_{0}\left[1-X_{2}(0)\right]}\right) \alpha_{1}^{k_{1}}, k_{1}=1, \ldots, N_{1}, \\
& X_{2}\left(k_{2}\right)=X_{2}(0)\left(\frac{1}{1-p_{0}\left[1-X_{1}(0)\right]}\right) \alpha_{2}^{k_{2}}, k_{2}=1, \ldots, N_{2},
\end{aligned}
$$

where $X_{1}(0), X_{2}(0)$ are the steady states of (3.3) and

$\alpha_{1}=\frac{p_{1}\left(1-p_{0}\left[1-\mathrm{X}_{2}(0)\right]\right)}{p_{0}\left[1-\mathrm{X}_{2}(0)\right]\left(1-p_{1}\right)}, \alpha_{2}=\frac{p_{2}\left(1-p_{0}\left[1-\mathrm{X}_{1}+(0)\right]\right)}{p_{0}\left[1-\mathrm{X}_{1}(0)\right]\left(1-p_{2}\right)}$.

Corollary 3.2: For the assembly system defined by assumptions (i)-(vi),

$$
\tilde{X}_{i}\left(k_{i}\right)=X_{i}\left(k_{i}\right)+\mathcal{O}(\mu), k_{i}=1, \ldots, N_{i}, \quad i=1,2 .
$$

Proof: See Appendix A.

Corollary 3.3: The calculated production rate, $\operatorname{PR}\left(p_{1}, p_{2}, p_{0}, N_{1}, N_{2}\right)$, defined by (3.3), (3.5), is a monotonically increasing function of all its arguments.

Proof: See Appendix A.

The monotonicity property of the assembly system cited above is quite similar to that established in [14]-[15].

\section{IMPROVABILITY WITH RESPECT TO WF}

\subsection{Improvability Conditions}

In terms of the calculated production rate, assembly system (i)-(vi) is improvable with respect to $\mathrm{WF}$ if there exist $p_{0}^{*}, p_{1}^{*}, p_{2}^{*}$ such that $p_{1}^{*} p_{2}^{*} p_{0}^{*}=p^{*}$ and 


$$
P R\left(p_{1}^{*}, p_{2}^{*}, p_{0}^{*}, N_{1}, N_{2}\right)>P R\left(p_{1}, p_{2}, p_{0}, N_{1}, N_{2}\right) .
$$

THEOREM 4.1: Assembly system (i)-(vi) is unimprovable with respect to $W F$ if and only if

$$
\begin{aligned}
& p_{1}=p_{0}\left[1-X_{2}(0)\right], \\
& p_{2}=p_{0}\left[1-X_{1}(0)\right] .
\end{aligned}
$$

where $X_{i}(0), i=1,2$, is the steady state of the recursive procedure (3.3). Proof: See Appendix B.

To reformulate condition (4.1) in terms of the data available on the factory floor, let $\widetilde{c b}_{i}, i=1,2$, denote the probability of the so-called communication blockage of the component machine $i$ :

$$
\widetilde{c b}_{i}=\operatorname{Prob}\left\{b_{i} \text { is full and } m_{0} \text { fails to take parts from } b_{i}\right\}, i=1,2
$$

Let $\widetilde{c s}_{i}, i=1,2$, denote the probability of the communication starvation of the assembly machine $m_{0}$ due to the lack of parts produced by the component machine $i$ :

$$
\widetilde{c s_{i}}=\operatorname{Prob}\left\{b_{i} \text { is empty }\right\}, i=1,2 .
$$

Finally, let $\overline{\tilde{h}}_{i}, i=1,2$, denote the average steady state occupancy of buffer $b_{i}$.

Corollary 4.1: Under condition (4.1),

(a).

$$
\widetilde{c b}_{i}=\widetilde{c s}_{i}+\mathscr{O}(\mu), i=1,2
$$

(b).

$$
\tilde{\tilde{h}}_{i}=E\left[\tilde{h}_{i}\right]=\frac{N_{i}}{2} \frac{N_{i}+1}{N_{i}+1-p_{i}}+\mathcal{O}(\mu) \approx \frac{N_{i}}{2}, i=1,2 .
$$


Proof: See Appendix B.

Since, as it is stated in Corollaries 3.1 and 3.2, the exact and the calculated production rates and buffer occupancies are $O(\mu)$ close to each other, from Corollary 4.1 follows

IMPROVABILITY INDICATOR 4.1: Assembly system (i)-(vi) is unimprovable with respect to WF if the frequency of communication blockage of a component machine is close to the frequency of communication starvation of the assembly machine caused by the lack of parts produced by this component machine; when this situation takes place, each buffer is on the average almost half full.

Remark 4.1: Improvability indicator 4.1 can be rationalized as follows: Buffer $b_{1}$ protects $m_{1}$ from blockages and $m_{0}$ from starvations due to parts produced by $m_{1}$. From the point of view of $m_{1}$, buffer $b_{1}$ should be mostly empty; from the point of view of $m_{0}$, buffer $b_{1}$ should be mostly full. The compromise is: buffer half full or blockages equal starvations. This is accomplished by distributing $p^{*}$ in an unimprovable manner.

\subsection{Unimprovable Distribution of $\boldsymbol{p}^{\star}$}

Using improvability indicator 4.1 , one could determine how good the assembly system is with regard to WF distribution. This does not require the knowledge of the machines and buffers parameters, $p_{i}$ 's and $N_{i}$ 's. If, however, $N_{i}$ 's are known, the unimprovable value of $p_{i}$ 's can be calculated as follows:

Let $P R^{*}$ denote the production rate of the system under the best possible distribution of $p_{i}$ 's, i.e.,

$$
P R^{*}=\max _{p_{1} p_{2} p_{0}=p^{*}} P R\left(p_{1}, p_{2}, p_{0}, N_{1}, N_{2}\right)
$$

Introduce the recursive procedure:

$$
x(s+1)=\left(p^{*}\right)^{\frac{1}{3}}\left(\frac{N_{1}+x(s)}{N_{1}+1}\right)^{\frac{2}{3}}\left(\frac{N_{2}+x(s)}{N_{2}+1}\right)^{\frac{2}{3}} .
$$


THEOREM 4.2: Assume $\frac{1}{N_{1}}+\frac{1}{N_{2}} \leq \frac{3}{2}$. Then the recursive procedure (4.3) is a contraction on $[0,1]$. Moreover,

$$
\lim _{s \rightarrow \infty} x(s)=P R^{*}
$$

where $P R^{*}$ is defined by (4.2). In addition, the values of $p_{i}, i=0,1,2$, that result in $P R^{*}$ are:

$$
\begin{gathered}
p_{i}^{*}=\left(\frac{N_{i}+1}{N_{i}+P R^{*}}\right) P R^{*}, i=1,2, \\
p_{0}^{*}=\left(\frac{N_{1}+1}{N_{1}+P R^{*}}\right)\left(\frac{N_{2}+1}{N_{2}+P R^{*}}\right) P R^{*} .
\end{gathered}
$$

Proof: See Appendix B.

Remark 4.2: Since, as it follows from the above $p_{0}^{*} / p_{i}^{*}>1$, Theorem 4.2 confirms the conclusion, established in [12], concerning the bowl phenomenon for the assembly systems.

\section{IMPROVABILITY WITH RESPECT TO WF AND WIP SIMULTANEOUSLY}

\subsection{Improvability Conditions}

In terms of the calculated production rate, the assembly system (i)-(vi) is improvable with respect to WF and WIP simultaneously if there exist $p_{0}^{*}, p_{1}^{*}, p_{2}^{*}$ and $N_{1}^{*}, N_{2}^{*}$ such that $p_{1}^{*} p_{2}^{*} p_{0}^{*}=p^{*}, N_{1}^{*}+N_{2}^{*}=N^{*}$ and

$$
P R\left(p_{1}^{*}, p_{2}^{*}, p_{0}^{*}, N_{1}^{*}, N_{2}^{*}\right)>P R\left(p_{1}, p_{2}, p_{0}, N_{1}, N_{2}\right) .
$$

THEOREM 5.1: Assembly system (i)-(vi) is unimprovable with respect to WF and WIP simultaneously if and only if (4.1) takes place and, in addition,

$$
X_{1}(0)=X_{2}(0) .
$$


where, as before, $X_{i}(0), i=1,2$, is the steady state of the recursive procedure (3.3).

Proof: See Appendix C.

Corollary 5.1: Under conditions (4.1) and (5.1),

$$
\overline{\tilde{h}}_{1}=\overline{\tilde{h}}_{2}+O(\mu)
$$

where $\overline{\tilde{h}}_{i}, i=1,2$, is the average steady state occupancy of buffer $b_{i}$.

Proof: See Appendix C.

From Corollaries 4.1 and 5.1 follows

IMPROVABILITY INDICATOR 5.1: Assembly system (i)-(vi) is unimprovable with respect to WF and WIP simultaneously if each buffer is on the average close to being half full and the average occupancy of each buffer is almost the same.

Remark 5.1: A rationalization of Improvability indicator 5.1 can be given as follows: Buffers $b_{1}$ and $b_{2}$ protect $m_{0}$ from starvations for parts produced by $m_{1}$ and $m_{2}$, respectively. When $\overline{\tilde{h}}_{1}>\overline{\tilde{h}}_{2}$, a unit of the capacity of $b_{1}$ can be reallocated to $b_{2}$ so that the starvation of $m_{0}$ for parts produced by $m_{2}$ is reduced and, consequently, $P R$ is increased. Only when $\overline{\tilde{h}}_{1} \approx \overline{\tilde{h}}_{2}$, machine $m_{0}$ is protected equally from the starvations for each part type and, therefore, the system is unimprovable.

\subsection{Unimprovable Distribution of $\mathrm{p}^{\star}$ and $\mathrm{N}^{*}$}

The values of $p_{i}^{*}, i=0,1,2$, and $N_{j}^{*}, j=1,2$, which render the system unimprovable with respect to WF and WIP can be calculated as follows:

Theorem 5.2: Assume that $N^{*}$ is even and denote

$$
P R^{* *}=\max _{\substack{p_{1}, p_{2}, p_{0} ; p_{1} p_{2} p_{0}=p^{*} \\ N_{1}, N_{2} ; N_{1}+N_{2}=N^{*}}} P R\left(p_{1}, p_{2}, p_{0}, N_{1}, N_{2}\right) .
$$

Then conditions (4.1) and (5.1) are satisfied if and only if, 


$$
\begin{aligned}
& p_{i}^{*}=\left(\frac{\frac{N^{*}}{2}+1}{\frac{N^{*}}{2}+P R^{*}}\right) P R^{*}, i=1,2, \\
& p_{0}^{*}=\left(\frac{\frac{N^{*}}{2}+1}{\frac{N^{*}}{2}+P R^{*}}\right)^{2} P R^{*}, \\
& N_{i}^{*}=\frac{N^{*}}{2}
\end{aligned}
$$

where $P R^{* *}$ is calculated according to recursive procedure (4.3) with $N_{i}$ 's defined by (5.3).

Proof: See Appendix C.

Thus, in an assembly system unimprovable with respect to both WF and WIP buffers $b_{1}$ and $b_{2}$ are of equal capacity and $p_{1}=p_{2}<p_{0}$, as indicated in (5.3).

\section{IMPROVABILITY WITH RESPECT TO WIP}

In terms of the calculated production rate, assembly system (i)-(vi) is improvable with respect to WIP if there exist $N_{1}^{*}$ and $N_{2}^{*}$ such that $N_{1}^{*}+$ $N_{2}^{*}=N^{*}$ and

$$
P R\left(p_{1}, p_{2}, p_{0}, N_{1}^{*}, N_{2}^{*}\right)>P R\left(p_{1}, p_{2}, p_{0}, N_{1}, N_{2}\right)
$$

Unfortunately, no simple analytical criteria for improvability with respect to WIP have been found. However, motivated by condition (5.2), we formulate the rule given below and justify it by numerical analysis of the Markov chain.

IMPROVABILITY INDICATOR 6.1: Assembly system (i)-(vi) is unimprovable with respect to WIP if the average occupancy of $b_{1}$ is as close as possible 
to that of $b_{2}$, i.e., $\left|\tilde{\tilde{h}}_{1}-\overline{\tilde{h}}_{2}\right|$ is minimized over all $N_{1}$ and $N_{2}$ such that $N_{1}$ $+N_{2}=N^{*}$.

Remark 6.1: Recall that sign " $"$ indicates that $\tilde{\tilde{h}}_{i}$ is the exact (rather than calculated $h_{i}$ ) occupancy of buffer $b_{i}, i=1,2$.

Numerical Justification: For all systems considered, transition matrices have been constructed, stationary probabilities of all states have been calculated, and the average occupancies of buffers have been evaluated. A typical example is shown in Table II. Obviously, max $\widetilde{P R}$ takes place if $\left|\overline{\tilde{h}}_{1}-\overline{\tilde{h}}_{2}\right|$ is minimized. Similar results have been obtained in the majority of cases analyzed. Several counterexamples, however, have also been found. One of them is shown in Table III. Although in this example min I $\overline{\tilde{h}}_{1}-\overline{\tilde{h}}_{2} \mid$ does not result in the max $\widetilde{P R}$, the difference between max $\widetilde{P R}$ and the $\widetilde{P R}$ corresponding to $\min \left|\overline{\tilde{h}}_{1}-\overline{\tilde{h}}_{2}\right|$ is quite small. A similar situation was observed in every case where the above rule failed. Therefore, we conclude that Improvability indicator 6.1 results in either the best or "almost" the best WIP distribution.

Remark 6.2: The unimprovable distribution of $N_{1}$ and $N_{2}$ can be reached not only through the complete search as in Tables II and III but also using the following Algorithm: Estimate $\overline{\tilde{h}}_{1}-\overline{\tilde{h}}_{2}$; re-allocate a unit of the buffer capacity from $N_{1}$ to $N_{2}$ if this difference is positive and from $N_{2}$ to $N_{1}$ if it is negative; continue this process until a periodic cycle of length 2 is reached; choose $N_{1}$ and $N_{2}$ from the values on the cycle that give the largest $\widetilde{P R}$; stop. The performance of this algorithm in comparison with the complete search procedure is illustrated in Table IV: In all case analyzed, it resulted in the same distribution of $N_{i}$ 's as the complete search approach.

TABLE II Illustration of Improvability indicator $6.1\left(p_{1}=0.8, p_{2}=0.5, p_{0}=0.5, N_{1}+\right.$ $N_{2}=6$ )

\begin{tabular}{lccccc}
\hline$N_{1}$ & $N_{2}$ & $\overline{\tilde{h}}_{1}$ & $\overline{\tilde{h}}_{2}$ & $\left|\overline{\tilde{h}}_{1}-\overline{\tilde{h}}_{2}\right|$ & $\widetilde{P R}$ \\
\hline 5 & 1 & 4.8746 & 0.6667 & 4.2079 & 0.3333 \\
4 & 2 & 3.8177 & 1.2002 & 2.6175 & 0.4 \\
3 & 3 & 2.7891 & 1.7191 & 1.07 & 0.4281 \\
2 & 4 & 1.7987 & 2.285 & 0.4863 & 0.4406 \\
1 & 5 & 0.8938 & 3.2781 & 2.3843 & 0.4249 \\
\hline
\end{tabular}


TABLE III Counterexample for Improvability indicator $6.1\left(p_{1}=0.7, p_{2}=0.6, p_{0}=0.8\right.$, $N_{1}+N_{2}=6$ )

\begin{tabular}{lccccc}
\hline$N_{1}$ & $N_{2}$ & $\overline{\tilde{h}}_{1}$ & $\overline{\tilde{h}}_{2}$ & $\left|\overline{\tilde{h}}_{1}-\overline{\tilde{h}}_{2}\right|$ & $\widetilde{\mathrm{PR}}$ \\
\hline 5 & 1 & 4.2565 & 0.6534 & 3.6031 & 0.5199 \\
4 & 2 & 3.0152 & 0.9526 & 2.0626 & 0.5692 \\
3 & 3 & 2.0881 & 1.2351 & 0.853 & 0.5796 \\
2 & 4 & 1.3521 & 1.6724 & 0.3203 & 0.5792 \\
1 & 5 & 0.7607 & 2.7529 & 1.9922 & 0.5583 \\
\hline
\end{tabular}

TABLE IV Implementation of Improvability indicator 6.1 based on the complete search and the Algorithm $\left(N_{1}+N_{2}=6\right)$

\begin{tabular}{lcccccc}
\hline & & & \multicolumn{2}{c}{ Algothm } & \multicolumn{2}{c}{ Complete search } \\
\cline { 3 - 6 }$p_{1}$ & $p_{2}$ & $p_{0}$ & $N_{1}$ & $N_{2}$ & $N_{1}$ & $N_{2}$ \\
\hline 0.7 & 0.6 & 0.5 & 3 & 3 & 3 & 3 \\
0.7 & 0.6 & 0.65 & 3 & 3 & 3 & 3 \\
0.7 & 0.6 & 0.8 & 3 & 3 & 3 & 3 \\
0.8 & 0.5 & 0.5 & 2 & 4 & 2 & 4 \\
0.8 & 0.5 & 0.65 & 2 & 4 & 2 & 4 \\
0.8 & 0.5 & 0.8 & 2 & 4 & 2 & 4 \\
\hline
\end{tabular}

Remark 6.3: In the case of improvability with respect to WF and WIP simultaneously, the exact equality of the average buffer occupancies is possible due to the infinitesimal nature of the distribution of $p_{i}$ 's; this counteracts the discrete nature of the distribution of $N_{i}$ 's. Since in the case of improvability with respect to WIP only the $p_{i}$ 's are fixed, no equality of $\overline{\tilde{h}}_{1}$ and $\overline{\tilde{h}}_{2}$ can, in general, be reached. Only the minimization of the difference $\left|\overline{\tilde{h}}_{1}-\overline{\tilde{h}}_{2}\right|$ could be achieved, and the $N_{i}$ 's that result in this minimization are, according to the Improvability indicator 6.1 , the unimprovable values of WIP distribution.

\section{UNCONSTRAINED IMPROVABILITY: WF UNIMPROVABLE SYSTEMS}

According to Definition 2.4, $m_{i}$ is the bottleneck of the assembly system if

$$
\frac{\partial P R}{\partial p_{i}}>\frac{\partial P R}{\partial p_{j}}, \forall j \neq i
$$


As it has been pointed out in Section 2, a machine with the smallest $p_{i}$ is not necessarily the bottleneck. An example is given in Figure 3, where $m_{2}$ has the smallest $p_{i}$ but $m_{1}$ has the largest $\partial P R / \partial p_{i}$. In Figure 3 and all the subsequent figures, the numbers in the circles and the rectangles represent the machines' and buffers' parameters, $p_{i}$ and $N_{i}$, respectively, and the numbers under the machines are the estimates of $\partial P R / \partial p_{i}$ denoted as $\Delta \widetilde{P R} / \Delta p_{i}$ and calculated using the numerical Markov chain analysis.

It turns out, however, that if the assembly is unimprovable with respect to WF, the machine with the smallest $p_{i}$ is indeed the bottleneck. This follows from

THEOREM 7.1: If assembly system (i)-(vi) is unimprovable with respect to $W F$, then

$$
p_{1} \frac{\partial P R}{\partial p_{1}}=p_{2} \frac{\partial P R}{\partial p_{2}}=p_{0} \frac{\partial P R}{\partial p_{0}} .
$$

Proof: See Appendix D.

Equalities (7.1) imply that the smallest $p_{i}$ has the largest $\partial P R / \partial p_{i}$. Therefore,

IMPROVABILITY INDICATOR 7.1: To expose the bottleneck machine, first ensure that the system is unimprovable with respect to WF; then, the machine with the smallest production rate in isolation is the bottleneck.

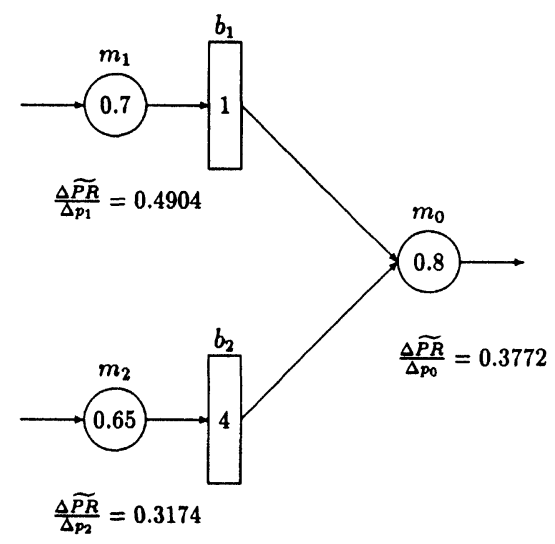

FIGURE 3 The bottleneck example. 


\section{UNCONSTRAINED IMPROVABILITY: THE GENERAL CASE}

\subsection{Preliminaries}

Most production systems on the factory floor are, unfortunately, improvable and often cannot be made unimprovable due to practical limitations. Therefore, it is of importance to identify bottlenecks in improvable systems. In many cases this is the only way for continuous improvements. This section is devoted to this topic.

Generally speaking, bottleneck machine of any assembly, improvable or not, can be determined by calculating $\partial \widetilde{P R} / \partial p_{i}$ and selecting $m_{i}$ corresponding to its largest value. Unfortunately, however, the calculation of $\partial \widetilde{P R} / \partial p_{i}$ is a difficult, if not an impossible, proposition. As it has been pointed out above, in the case of unimprovable systems this calculation is unnecessary: the slowest machine is always the bottleneck. Is there a way to identify bottlenecks in improvable systems without calculating $\partial \widetilde{P R} / \partial p_{i}$ ? It turns out that the answer is positive, and a way for such an identification is described below.

Let $\widetilde{m b}_{i}$ and $\widetilde{m s}_{i}$ denote the probabilities of the manufacturing blockage of the component machine $i, i=1,2$, and the manufacturing starvation of the assembly machine $m_{0}$ due to the lack of parts produced by the component machine $i, i=1,2$, respectively, i.e.,

$$
\widetilde{m b}_{i}=\operatorname{Prob}\left(\left\{m_{\mathrm{i}} \text { is up during a time slot }\right\}\right.
$$

$\cap\left\{b_{i}\right.$ is full at the beginning of this slot $\}$

$\cap\left\{m_{0}\right.$ fails to take a part from $b_{\mathrm{i}}$ at the beginning of this slot $\left.\}\right), i=1,2$,

$$
\widetilde{m s}_{i}=\operatorname{Prob}\left(\left\{m_{0} \text { is up during a time slot }\right\}\right.
$$

$\cap\left\{b_{i}\right.$ is empty at the beginning of this slot $\left.\}\right), i=1,2$.

We show below that bottlenecks in improvable systems can be identified by analyzing the relationships between the values $\widetilde{m b}_{i}$ and $\widetilde{m s}_{i}$ for each pair of consecutive machines. 


\subsection{The Case of a Single Bottleneck}

Consider an assembly shown in Figure 4(a). The two numbers under each component machine represent $\widetilde{m b}_{i}$ and an estimate of $\partial \widetilde{P R} / \partial p_{i}$ denoted as $\Delta \widetilde{P R} / \Delta p_{i}$. The assembly machine has three numbers, with $\widetilde{m s_{1}}$ and $\widetilde{m s_{2}}$ representing the probability of starvations due to the lack of part in $b_{1}$ and $b_{2}$, respectively, and $\Delta \widetilde{P R} / \Delta p_{0}$. Obviously, $m_{1}$ and $m_{2}$ are never starved and $m_{0}$ is never blocked; this is not represented in Figure 4. To identify the bottleneck machine, place arrows directed from one machine to another according to the following:
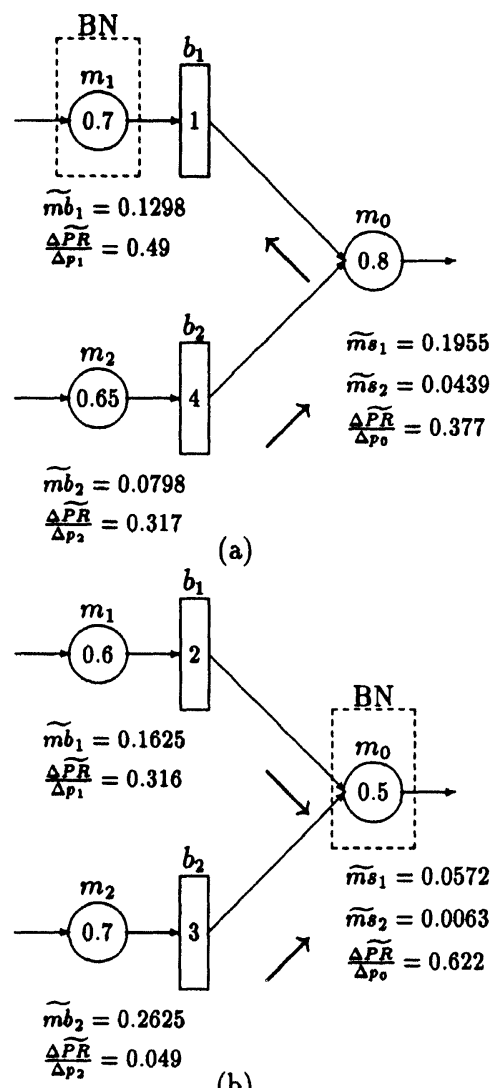

(b)

FIGURE 4 Illustration of Improvability indicator 8.1. 
RULE 8.1: If $\widetilde{m b}_{i}>\widetilde{m s}_{i}$, the arrow is directed from the upstream to the downstream machine; if $\widetilde{m b}_{i}<\widetilde{m s}_{i}$, the direction of the arrow is reversed.

The following improvability indicator identifies the bottleneck machine if it is unique. The case of multiple bottlenecks is addressed in Subsection 8.3 .

IMPROVABILITY INDICATOR 8.1: Assume that assembly system (i)-(vi) has a unique machine which has no arrows emanating from it. Then this machine is the bottleneck.

Thus, according to this indicator, the bottleneck of an assembly can be identified without calculating $\partial \widetilde{P R} / \partial p_{i}$, or even without the knowledge of the machines and buffers parameters, $p_{i}$ 's and $N_{i}$ 's, but just by observing the frequencies of blockages and starvations of the machines. Since these frequencies often are or can be measured on the factory floor, this indicator offers a constructive way for bottleneck identification.

Improvability indicator 8.1 is justified below both numerically and analytically.

Numerical Justification: The transition matrix for the Markovian chain defined by assumptions (i)-(vi) has been constructed and iterated upon to obtain stationary probability distributions for $p_{i}$ and $p_{i}+\Delta p_{i}$, with the step $\Delta p_{i}=0.01$. Then, the difference between the resulting production rates, $\Delta \widetilde{P R}$, has been calculated and $\Delta \widetilde{P R} / \Delta p_{i}$ has been evaluated. Two typical examples are shown in Figure 4. According to Improvability indicator 8.1, the bottleneck is the machine without arrows emanating from it. Hence, the bottlenecks in Figure 4(a) and (b) are component machine $m_{1}$ and assembly machine $m_{0}$, respectively. This conclusion is supported by the values of $\Delta \widetilde{P R} / \Delta p_{i}$.

For most systems considered the bottleneck identified using Improvability indicator 8.1 and $\Delta \widetilde{P R} / \Delta p_{i}$ coincides. An example in which the two results differ is shown in Figure 5: According to Improvability indicator 8.1 , the bottleneck is $m_{0}$ whereas according to $\Delta \widetilde{P R} / \Delta p_{i}$ the bottleneck is $m_{1}$. However, as it can be seen in Figure 5, the difference between $\Delta \widetilde{P R} / \Delta p_{1}$ and $\Delta \widetilde{P R} / \Delta p_{0}$ is quite small. The same situation has been observed in all counterexamples discovered. Therefore, we conclude that Improvability indicator 8.1 identifies either the machine with the largest $\partial$ $\widetilde{P R} / \partial p_{i}$ or a machine with $\partial \widetilde{P R} / \partial p_{i}$ being close to the largest one. 


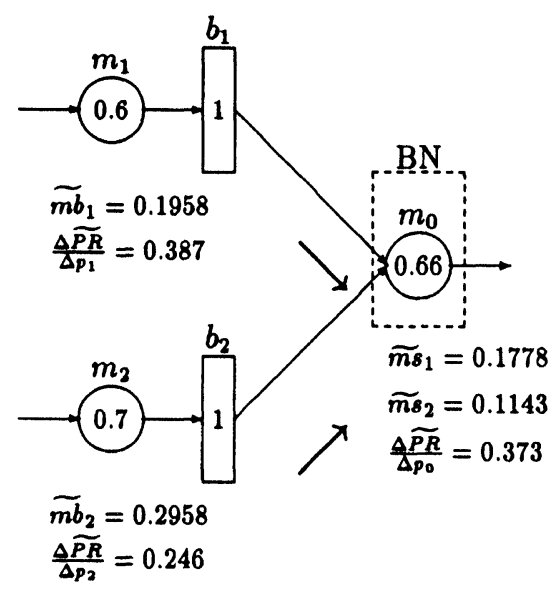

FIGURE 5 Counterexample for Improvability indicator 8.1.

Analytical Justification: An additional assumption is needed to carry out the analytical proof:

Hypothesis 8.1: In assembly system (i)-(vi), if

$$
m b_{j}>m s_{j}, j=1,2
$$

then

$$
X_{j}(0) \approx Q\left(p_{j}, p_{0}\left[1-X_{3-j}(0)\right], N_{j}\right)=: \epsilon_{j 1}<<1
$$

if

$$
m b_{j}<m s_{j}, j=1,2
$$

then

$$
\left(1-p_{0}\left[1-X_{3-j}(0)\right]\right) X_{j}\left(N_{j}\right) \approx Q\left(p_{0}\left[1-X_{3-j}(0)\right], p_{j}, N_{j}\right)=: \epsilon_{j 2}<<1 .
$$

Here $X_{j}(0)$ and $X_{j}\left(N_{j}\right)$ are the stationary probabilities that buffer $b_{j}, j=1,2$, is empty and full respectively, and function $Q(x, y, N)$ is defined in (3.3).

Expression (8.1) implies that if a component machine is blocked more often than the assembly machine is starved due to parts produced by this component machine, the probability that the buffer separating these two 
machines, is empty is small. Similarly, (8.2) implies that if a component machine is blocked less frequently than the assembly machine is starved, the probability that the buffers is full is small. Hypothesis 8.1 has been formulated based on numerical evidence obtained in simulations.

THEOREM 8.1: Assume that system (i)-(vi) has a unique machine $m_{i}, i \epsilon$ $\{0,1,2\}$, with no arrows emanating from it. Then, under Hypothesis 8.1, $m_{i}$ is the bottleneck in the sense that

$$
\frac{\partial P R}{\partial p_{i}}>\frac{\partial P R}{\partial p_{j}}, \forall j \neq i
$$

Proof: See Appendix E.

\subsection{Multiple Bottlenecks}

Assume now that the assembly system has two machines with no arrows emanating from them. Under the Rule 8.1, these could be only the two component machines. Which one, then, is the bottleneck? To answer this question, introduce the notion of a bottleneck severity:

$$
\tilde{S}_{i}=\widetilde{m s}_{i}-\widetilde{m b}_{i}, i=1,2
$$

IMPROVABILITY INDICATOR 8.2: If the assembly system (i)-(vi) has both component machines with no arrows emanating from them, the one with the largest severity is the primary bottleneck, i.e. defines the largest $\partial \widetilde{P R} / \partial p_{i}$.

Numerical Justification: Improvability indicator 8.2 has also been justified using the direct numerical Markov chain analysis. Two typical examples are shown in Figure 6, where the last row of numbers is the bottleneck severity. In most cases analyzed, Improvability indicator 8.2 resulted in the correct identification of the primary bottleneck. A number of counterexamples have also been found. One of them is shown in Figure 7. Nevertheless, since in the majority of cases analyzed Improvability indicator 8.2 identified the machine with the best possible effect on the production rate, we conclude that it can be employed as a tool for the primary bottleneck identification. 


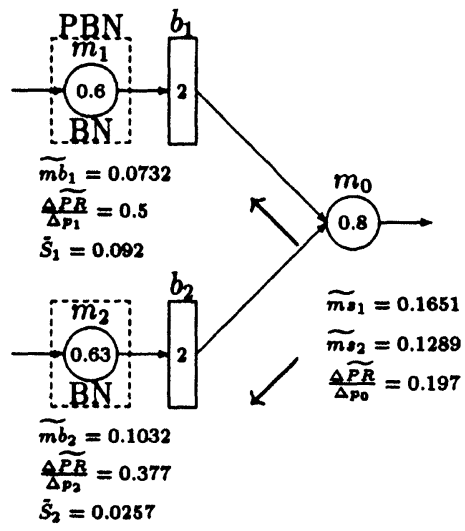

(a)

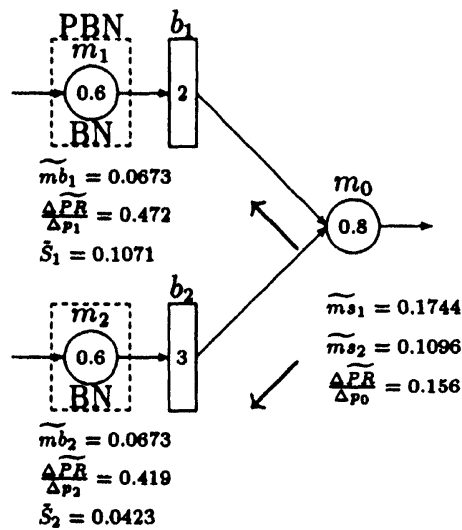

(b)

FIGURE 6 Illustration of Improvability indicator 8.2.

\section{THE CASE OF $n$ COMPONENT-ONE ASSEMBLY MACHINE SYSTEM}

Results described in Sections 3-8 can be generalized for the case of the assembly that consists on $n$ component machines working in parallel and one assembly machine Figure 8 ). Such a system is defined by assumptions (i)-(vi) with obvious changes pertaining to the number of the component machines (see Remark 2.1). The notions of improvability also remain the same with obvious changes in Definitions 2.1-2.5 (Remark 2.7). Below, we 


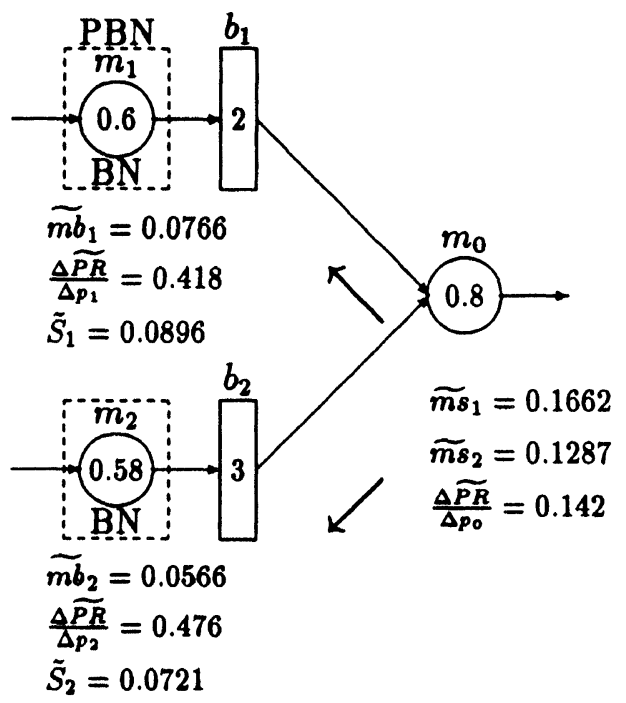

FIGURE 7 Counterexample for Improvability indicator 8.2.

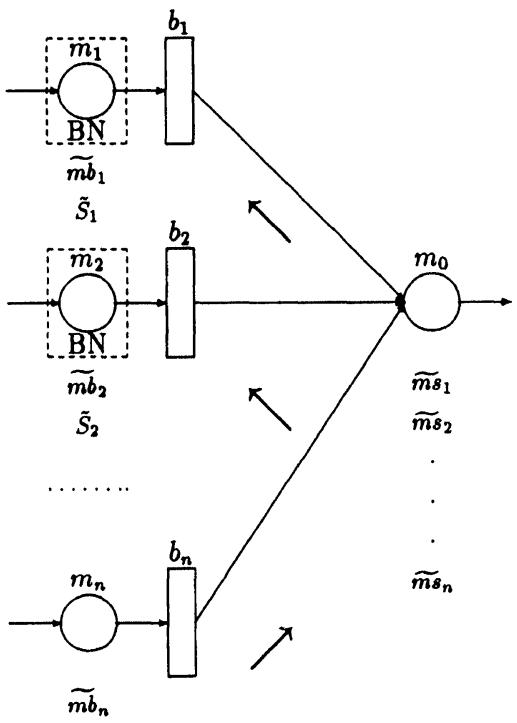

FIGURE $8 \quad n$ component-one assembly machine system.

cite main results on improvability of such a system. The proofs are straightforward generalizations of those given in Appendices A-E and are not included here. 


\subsection{Production Rate Evaluation}

Using arguments similar to those described in Section 3, the production rate, $\widetilde{P R}$, of the assembly systems with $n$ component machines is represented as

$$
\widetilde{P R}=p_{0} \prod_{i=1}^{n}\left[1-\tilde{X}_{i}(0)\right]+\mathcal{O}(\mu)
$$

where $p_{0}$ is the isolation production rate of the assembly machine, $\tilde{X}_{i}(0)$, $i=1, \ldots, n$, is the steady state probability of buffer $i$ being empty, and $\mu \ll 1$. The analytical approximations of $\tilde{X}_{i}(0)$ are obtained through the following recursive procedure:

$$
\begin{gathered}
X_{i}(0, s+1)=Q\left(p_{1}, p_{0} \prod_{j=1}^{i-1}\left[1-X_{j}(0, s+1)\right] \prod_{j=i+1}^{n}\left[1-X_{j}(0, s)\right], N_{i}\right), \\
i=1, \ldots, n, \\
X_{i}(0,0)=0, \quad i=2, \ldots, n, \quad s=0,1,2, \ldots
\end{gathered}
$$

Lemma 9.1: Procedure (9.1) is convergent, i.e., the following limits exist:

$$
\lim _{s \rightarrow \infty} X_{i}(0, s)=: X_{i}(0), \quad i=1, \ldots, n .
$$

THEOREM 9.1: For the assembly system with $n$ component machines,

$$
\tilde{X}_{i}(0)=X_{i}(0)+\mathscr{O}(\mu), \quad i=1, \ldots, n .
$$

As before, define the calculated production rate as follows

$$
P R=p_{0} \prod_{i=1}^{n}\left[1-X_{i}(0)\right]
$$

where $X_{i}(0), i=1, \ldots, n$, are the steady states of (9.1).

Corollary 9.1:

$$
\widetilde{P R}=P R+O(\mu) .
$$


Corollary 9.2: The calculated production rate, $P R\left(p_{1}, \ldots, p_{n}, p_{0}, N_{1}, \ldots\right.$, $N_{n}$ ), defined by (9.2) is a monotonically increasing function of all its arguments.

\subsection{Constrained Improvability}

\subsubsection{WF improvability}

THEOREM 9.2: Assembly system with $n$ component machines is unimprovable with respect to WF if and only if

$$
p_{i}=p_{0} \prod_{j=1}^{i-1}\left[1-X_{j}(0)\right] \prod_{j=i+1}^{n}\left[1-X_{j}(0)\right], \quad i=1, \ldots, n .
$$

Corollary 9.3: Under conditions (9.3),

(a).

$$
\widetilde{c b}_{i}=\widetilde{c s}_{i}+\mathcal{O}(\mu), \quad i=1, \ldots, n
$$

(b).

$$
\overline{\tilde{h}}_{i}=E\left[\tilde{h}_{i}\right]=\frac{N_{i}}{2} \frac{N_{i}+1}{N_{i}+1-p_{i}}+\mathcal{O}(\mu) \approx \frac{N_{i}}{2}, \quad i=1, \ldots, n,
$$

where $\widetilde{c b}_{i}$ and $\widetilde{c s}_{i}$ are the probabilities of communication blockage and starvation of machine $i$, respectively, and $\overline{\tilde{h}}_{i}$ is the average occupancy of buffer $i$.

Therefore,

IMPROVABILITY INDICATOR 9.1: Assembly system (i)-(vi) with $n$ component machines is unimprovable with respect to WF if the frequency of communication blockage of each component machine is close to the frequency of communication starvation of the assembly machine caused by the lack of the parts produced by this particular component machine; when this situation takes place, each buffer is on the average almost half full.

Unimprovable distribution of $\mathrm{p}_{\mathrm{i}}$ 's is given by 
THEOREM 9.3: Assume $\sum_{i=1}^{n} \frac{1}{N_{i}} \leq \frac{n+1}{2}$. Then

$$
\begin{gathered}
p_{i}^{*}=\left(\frac{N_{i}+1}{N_{i}+P R^{*}}\right) P R^{*}, i=1, \ldots, n, \\
p_{0}^{*}=\prod_{i=1}^{n}\left(\frac{N_{i}+1}{N_{i}+P R^{*}}\right) P R^{*} .
\end{gathered}
$$

where

$$
P R^{*}=\lim _{s \rightarrow \infty} x(s)
$$

and $x(s)$ is defined by the following convergent recursive procedure:

$$
x(s+1)=\left(p^{*}\right) \frac{1}{n+1} \prod_{i=1}^{n}\left(\frac{N_{i}+x(s)}{N_{i}+1}\right)^{\frac{2}{n+1}} .
$$

Remark 9.1: In comparison with the two component machine case, (9.4) result in a larger imbalance between the component and the assembly machine. This effect was discovered in [12].

\subsubsection{WF \& WIP improvability}

THEOREM 9.4: Assembly system with $n$ component machines is unimprovable with respect to WF and WIP simultaneously if and only if (9.3) takes place and, in addition,

$$
X_{1}(0)=X_{2}(0)=\ldots=X_{n}(0)
$$

where, as before, $X_{i}(0), i=1, \ldots, n$, is the steady state of the recursive procedure (9.1).

CoRollary 9.4: Under conditions (9.3) and (9.6),

$$
\overline{\tilde{h}}_{1}=\overline{\tilde{h}}_{2}+\sigma(\mu)=\ldots=\overline{\tilde{h}}_{n}+\mathcal{O}(\mu),
$$

where $\overline{\tilde{h}}_{i}, i=1, \ldots, n$, is the average steady state occupancy of buffer $b_{i}$. 
Therefore,

IMPROVABILITY INDICATOR 9.2: Assembly system (i)-(vi) with $n$ component machines is unimprovable with respect to WF and WIP simultaneously if each buffer is on the average close to being half full and the average occupancy of each buffer is almost the same.

The unimprovable distribution of $\mathrm{p}_{\mathrm{i}}$ 's and $\mathrm{N}_{\mathrm{i}}$ 's is given by

THEOREM 9.5: Assume that $N^{*}$ is a multiple of $n$. Then

$$
\begin{gathered}
p_{1}^{*}=p_{2}^{*}=\ldots=p_{n}^{*}=\left(\frac{N_{1}^{*}+1}{N_{1}^{*}+P R^{* *}}\right) P R^{* *}, \\
p_{0}^{*}=\left(\frac{N_{1}^{*}+1}{N_{1}^{*}+P R^{* *}}\right)^{n} P R^{* *}, \\
N_{i}^{*}=\frac{N^{*}}{n}, \quad i=1, \ldots, n,
\end{gathered}
$$

where $P R^{* *}$ is calculated according to the recursive procedure (9.5) with $N_{i}$ 's defined by (9.7).

\subsubsection{WIP improvability}

The following has been justified numerically:

IMPROVABILITy INDICATOR 9.3: Assembly system (i)-(vi) with $n$ component machines is unimprovable with respect to WIP if the average occupancies of all buffers are as close to each other as possible, i.e., $\max \left|\tilde{\tilde{h}}_{i}-\overline{\tilde{h}}_{j}\right|$ is minimized over all $N_{i}$ 's such that $\sum_{i=1}^{n} N_{i}=N^{*}$.

Remark 9.2: The unimprovable distribution of $N_{i}$ 's can be reached using an algorithm similar to that described in Remark 6.1. 


\subsection{Unconstrained Improvability}

\subsubsection{WF unimprovable case}

THEOREM 9.6: For assembly system (i)-(vi) with $n$ component machines, unimprovable with respect to $W F$,

$$
p_{1} \frac{\partial P R}{\partial p_{1}}=p_{2} \frac{\partial P R}{\partial p_{2}}=\ldots=p_{n} \frac{\partial P R}{\partial p_{n}}=p_{0} \frac{\partial P R}{\partial p_{0}}
$$

Equalities (9.8), obviously, imply that smallest $p_{i}$ has the largest $\partial P R /$ $\partial p_{i}$. Therefore,

IMPROVABILITY INDICATOR 9.4: To expose the bottleneck machine in a system with $n$ component machines, first ensure that it is unimprovable with respect to WF; then, the machine with the smallest $p_{i}$ is the bottleneck.

\subsubsection{Improvable case}

Consider an assembly shown in Figure 8. As in the two component machines case, the symbol $\widetilde{m b}_{i}$ under each component machine represents the probabilities of manufacturing blockage and the symbols, $\widetilde{m s}_{i}, i=1, \ldots, n$, under the assembly machine represent the probability of manufacturing starvation due to the lack of parts produced by $m_{i}$. The arrows are placed according to the Rule 8.1: If $\widetilde{m b}_{i}>\widetilde{m s}_{i}$, the arrow is directed from the upstream to the downstream machine; if $\widetilde{m b}_{i}<\widetilde{m s}_{i}$, the direction of the arrow is reversed. For each machine with no arrows emanating from it, the following value of severity is assigned:

$$
\widetilde{S}=\widetilde{m s}_{i}-\widetilde{m b}_{i}, i=1, \ldots, n
$$

IMPROVABILITY INDICATOR 9.5: If the assembly system (i)-(vi) with $n$ component machines has a single machine with no arrows emanating from it, then this machine is the bottleneck. If there are multiple machines with no emanating arrows, the primary bottleneck is that with the largest severity. 


\section{CONCLUSIONS}

An effective continuous improvement process is considered by many as a necessary condition to achieve and maintain a competitive advantage in today's manufacturing market place. Often, however, it is not obvious how to select, design, and implement a continuous improvement process which would be most effective in a given practical situation. Although the general philosophy of manufacturing gurus, such as Diming, Taguchi, Goldratt, is quite helpful, quantitative engineering techniques might also be productive in helping to design a continuous improvement project, in the same manner as products and processes are designed. The theory developed in this paper provides an instance of such a technique. The authors used it in five different continuous improvement projects carried out during the last 3 years at two automotive component plants and found it to be a useful tool for both improvement of existing production systems and design of new ones. The results of these applications will be reported elsewhere. It is hoped that this approach will prove to be useful for other practitioners and theoreticians alike.

\section{APPENDIX A. PROOFS FOR SECTION 3}

Proof of Lemma 3.1: By induction: Since function $Q(x, y, N)$ takes values in $(0,1)$ and is monotonically increasing in $y,[1]$, for $s=0$ we have

$$
\begin{aligned}
X_{1}(0,1) & =Q\left(p_{1}, p_{0}\left[1-X_{2}(0,0)\right], N_{1}\right) \\
& =Q\left(p_{1}, p_{0}, N_{1}\right) \\
& >Q\left(p_{1}, p_{0}\left[1-Q\left(p_{2}, p_{0}\left[1-X_{1}(0,1)\right], N_{2}\right)\right], N_{1}\right) \\
& =Q\left(p_{1}, p_{0}\left[1-X_{2}(0,1)\right], N_{1}\right) \\
& =X_{1}(0,2) .
\end{aligned}
$$

Assume that for $s>0$,

$$
X_{1}(0, s)<X_{1}(0, s-1) .
$$


Then, due to the monotonic properties of $Q(x, y, N)$,

$$
\begin{aligned}
X_{2}(0, s-1) & =Q\left(p_{2}, p_{0}\left[1-X_{1}(0, s-1)\right], N_{2}\right) \\
& <Q\left(p_{2}, p_{0}\left[1-X_{1}(0, s)\right], N_{2}\right) \\
& =X_{2}(0, s)
\end{aligned}
$$

and

$$
\begin{aligned}
X_{1}(0, s+1) & =Q\left(p_{1}, p_{0}\left[1-X_{2}(0, s)\right], N_{1}\right) \\
& <Q\left(p_{1}, p_{0}\left[1-X_{2}(0, s-1)\right], N_{1}\right) \\
& =X_{1}(0, s) .
\end{aligned}
$$

Thus, $X_{1}(0, s)$ is monotonically decreasing in $s$. Since the sequence $X_{1}(0, s)$ is monotonic and bounded from above and below (function $Q$ takes values in $(0,1))$, it is convergent, i.e.,

$$
\lim _{s \rightarrow \infty} X_{1}(0, s)=: X_{1}(0) .
$$

Similar arguments are used to prove that $X_{2}(0, s)$ is convergent and monotonically increasing.

To prove Theorem 3.1, we need the following 5 lemmas:

Lemma A.1: Let

$$
\begin{aligned}
& \tilde{p}_{0}^{1}:=\operatorname{Prob}\left\{\text { machine } m_{0} \text { produces } \mid \text { buffer } b_{1} \text { has parts }\right\} \\
& \tilde{p}_{0}^{2}:=\operatorname{Prob}\left\{\text { machine } m_{0} \text { produces } \mid \text { buffer } b_{2} \text { has parts }\right\}
\end{aligned}
$$

Then, under Numerical Fact 3.1,

$$
\begin{aligned}
& \tilde{p}_{0}^{1}=p_{0}\left[1-\tilde{X}_{2}(0)\right]+\mathcal{O}(\mu), \\
& \tilde{p}_{0}^{2}=p_{0}\left[1-\tilde{X}_{1}(0)\right]+\mathcal{O}(\mu),
\end{aligned}
$$


where $\tilde{X}_{1}(0)$ and $\tilde{X}_{2}(0)$ are the stationary probabilities that buffer $b_{1}$ and $b_{2}$ are empty.

Proof: Based on the conditional probability formula and Numerical Fact 3.1, we write:

$$
\begin{gathered}
\operatorname{Prob}\left\{b_{1} \text { is empty } \mid b_{2} \text { has parts }\right\} \\
=\frac{\sum_{k_{2}=1}^{N_{2}} \tilde{X}_{1,2}\left(0, k_{2}\right)}{\sum_{k_{2}=1}^{N_{2}} \tilde{X}_{2}\left(k_{2}\right)} \\
=\frac{\sum_{k_{2}=1}^{N_{2}} \tilde{X}_{1}(0) \tilde{X}_{2}\left(k_{2}\right)}{1-\tilde{X}_{2}(0)}+\mathcal{O}(\mu) \\
=\frac{\tilde{X}_{1}(0)\left[1-\tilde{X}_{2}(0)\right]}{1-\tilde{X}_{2}(0)}+\mathcal{O}(\mu) \\
=\tilde{X}_{1}(0)+\mathcal{O}(\mu) \\
=\operatorname{Prob}\left\{b_{1} \text { is empty }\right\}+\mathcal{O}(\mu) .
\end{gathered}
$$

Using repeatedly the conditional probability formula, the definition of $\tilde{p}_{0}^{1}$, and (A.5), we obtain:

$$
\begin{aligned}
& \tilde{p}_{0}^{1}= \operatorname{Prob}\left\{m_{0} \text { produces } \mid b_{1} \text { has parts }\right\} \\
&= \operatorname{Prob}\left\{m_{0} \text { is up, } b_{1} \text { has parts, and } b_{2} \text { has parts } \mid b_{1} \text { has parts }\right\} \\
&= \frac{\operatorname{Prob}\left\{m_{0} \text { is up, } b_{1} \text { has parts, and } b_{2} \text { has parts }\right\}}{\operatorname{Prob}\left\{b_{1} \text { has parts }\right\}} \\
&= \operatorname{Prob}\left\{m_{0} \text { is up } \mid b_{1} \text { has parts and } b_{2} \text { has parts }\right\} . \\
& \frac{\operatorname{Prob}\left\{b_{1} \text { has parts } \mid b_{2} \text { has parts }\right\}}{\operatorname{Prob}\left\{b_{1} \text { has parts }\right\}} \\
& \cdot \operatorname{Prob}\left\{b_{2} \text { has parts }\right\}
\end{aligned}
$$




$$
\begin{aligned}
& =p_{0}\left[1-\tilde{X}_{2}(0)\right] \frac{\operatorname{Prob}\left\{b_{1} \text { has parts } \mid b_{2} \text { has parts }\right\}}{\operatorname{Prob}\left\{b_{1} \text { has parts }\right\}} \\
& =p_{0}\left[1-\tilde{X}_{2}(0)\right] \frac{1-\operatorname{Prob}\left\{b_{1} \text { is empty } \mid b_{2} \text { has parts }\right\}}{1-\operatorname{Prob}\left\{b_{1} \text { is empty }\right\}} \\
& =p_{0}\left[1-\tilde{X}_{2}(0)\right]+O(\mu) .
\end{aligned}
$$

This proves the first statement of the lemma. The second is proved analogously.

Consider now 2 two-machine-one-buffer lines, $L_{1}$ and $L_{2}$, where the first machine of $L_{1}$ has the isolation production rate $p_{1}$, the second $\tilde{p}_{0}^{1}$, and the buffer capacity is $N_{1}$; the first machine of $L_{2}$ is characterized by $p_{2}$, the second by $\tilde{p}_{0}^{2}$, and the buffer capacity is $N_{2}$. Let $\bar{X}_{1}(\cdot)$ and $\bar{X}_{2}(\cdot)$ be the stationary probability distributions of the buffer occupancy of lines $L_{1}$ and $L_{2}$, respectively, and let $\tilde{X}_{1}(\cdot)$ and $\tilde{X}_{2}(\cdot)$ be the stationary probability distributions of the occupancy of beffers $b_{1}$ and $b_{2}$ in the assembly system (i)-(vi). Then, we have

LEMMA A.2: Under Numerical Fact 3.1, the following is true:

$$
\tilde{X}_{i}\left(k_{i}\right)=\bar{X}_{i}\left(k_{i}\right)+\mathcal{O}(\mu), k_{i}=0, \ldots, N_{i}, i=1,2 .
$$

Proof: Since assembly system (i)-(vi) can be described by an ergodic Markov chain with states $\tilde{\mathrm{X}}_{1,2}\left(k_{1}, k_{2}\right)$, in the steady state we write:

$$
\begin{aligned}
& \tilde{X}_{1,2}\left(0, k_{2}\right) \\
& =\sum_{k_{2}} \tilde{X}_{1,2}\left(0, k_{2}^{\prime}\right) \operatorname{Prob}\left\{m_{1} \text { does not produce } \mid 0, k_{2}^{\prime}\right\} \operatorname{Prob}\left\{k_{2}^{\prime} \rightarrow k_{2} \mid 0 \rightarrow 0\right\} \\
& +\sum_{k_{2}} \tilde{X}_{1,2}\left(1, k_{2}^{\prime}\right) \operatorname{Prob}\left\{m_{1} \text { does not produce, } m_{0} \text { produces } \mid 1, k_{2}^{\prime}\right\}
\end{aligned}
$$

$$
\operatorname{Prob}\left\{k_{2}^{\prime} \rightarrow k_{2} \mid 1 \rightarrow 0\right\}
$$


where $\operatorname{Prob}\left\{m_{1}\right.$ does not produce $\left.\mid k_{1}, k_{2}\right\}$ denotes the conditional probability that machine $m_{1}$ does not produce a part during a cycle, giventhat buffer $b_{1}$ contains $k_{1}$ and buffer $b_{2}$ contains $k_{2}$, and Prob $\left\{k_{2}^{\prime} \rightarrow k_{2} \mid k_{1}^{\prime} \rightarrow k_{1}\right\}$ denotes the conditional probability of the transition from the state where buffer $b_{2}$ contains $k_{2}^{\prime}$ parts, given that the number of parts in buffer $b_{1}$ change from $k_{1}^{\prime}$ to $k_{1}$. Summation over all $k_{2}$ yields

$$
\begin{aligned}
& \widetilde{X}_{1}(0) \\
= & \sum_{k_{2}^{\prime}} \tilde{X}_{1,2}\left(0, k_{2}^{\prime}\right) \operatorname{Prob}\left\{m_{1} \text { does not produce } \mid 0, k_{2}^{\prime}\right\} \sum_{k_{2}} \operatorname{Prob}\left\{k_{2}^{\prime} \rightarrow k_{2} \mid 0 \rightarrow 0\right\} \\
+ & \sum_{k_{2}^{\prime}} \tilde{X}_{1,2}\left(1, k_{2}^{\prime}\right) \operatorname{Prob}\left\{m_{1} \text { does not produce, } m_{0} \text { produces } \mid 1, k_{2}^{\prime}\right\} \\
& \sum_{k_{2}} \operatorname{Prob}\left\{k_{2}^{\prime} \rightarrow k_{2} \mid 1 \rightarrow 0\right\},
\end{aligned}
$$

SinceProb $\left\{k_{2}^{\prime} \rightarrow k_{2} \mid 0 \rightarrow 0\right\}=1$,

$$
\begin{aligned}
\tilde{X}_{1}(0) & =\sum_{k_{2}^{\prime}} \tilde{X}_{1,2}\left(0, k_{2}^{\prime}\right) \operatorname{Prob}\left\{m_{1} \text { does not produce } \mid 0, k_{2}^{\prime}\right\} \\
& +\sum_{k_{2}^{\prime}} \tilde{X}_{1,2}\left(1, k_{2}^{\prime}\right) \operatorname{Prob}\left\{m_{1} \text { does not produce, } m_{0} \text { produces } \mid 1, k_{2}^{\prime}\right\} .
\end{aligned}
$$

Consider the first term of the right hand side of equation (A.9). Since $m_{1}$ is the first machine and $b_{1}$ contains zero parts, $m_{1}$ is neither starved nor blocked. Therefore,

$$
\begin{aligned}
& \sum_{k_{2}^{\prime}} \tilde{X}_{1,2}\left(0, k_{2}^{\prime}\right) \operatorname{Prob}\left\{m_{1} \text { does not produce } \mid 0, k_{2}^{\prime}\right\} \\
& =\left(1-p_{1}\right) \sum_{k_{2}^{\prime}} \tilde{X}_{1,2}\left(0, k_{2}^{\prime}\right) \\
& =\left(1-p_{1}\right) \tilde{X}_{1}(0)
\end{aligned}
$$


For the second term of the right hand side of equation (A.9), we write:

$$
\begin{aligned}
& \sum_{k_{2}^{\prime}} \tilde{X}_{1,2}\left(1, k_{2}^{\prime}\right) \operatorname{Prob}\left\{m_{1} \text { does not produce, } m_{0} \text { produces } \mid 1, k_{2}^{\prime}\right\} \\
& =\sum_{k_{2}^{\prime} \geq 1} \tilde{X}_{1,2}\left(1, k_{2}^{\prime}\right) \operatorname{Prob}\left\{m_{1} \text { does not produce, } m_{0} \text { produces } \mid 1, k_{2}^{\prime}\right\} \\
& +\tilde{X}_{1,2}(1,0) \operatorname{Prob}\left\{m_{1} \text { does not produce, } m_{0} \text { produces } \mid 1,0\right\} .
\end{aligned}
$$

When $b_{1}$ contains one part and $b_{2}$ contains at least one part, $m_{0}$ is not starved. If $m_{0}$ produces a part, then $m_{1}$ is not blocked. Therefore, the conditional probability in the first term on the right hand side of equation (A.11) is equal to $\left(1-p_{1}\right) p_{0}$. When $b_{2}$ contains zero parts, $m_{0}$ is starved, and the conditional probability in the second term on the right hand side of equation (A.11) is equal to 0 . Thus,

$$
\begin{aligned}
& \sum_{k_{2}^{\prime}} \tilde{X}_{1,2}\left(1, k_{2}^{\prime}\right) \operatorname{Prob}\left\{m_{1} \text { does not produce, } m_{0} \text { produces } \mid 1, k_{2}^{\prime}\right\} \\
& =\left(1-p_{1}\right) p_{0} \sum_{k_{2}^{\prime} \geq 1} \tilde{X}_{1,2}\left(1, k_{2}^{\prime}\right)
\end{aligned}
$$

Under Numerical Fact 3.1, this can be rewritten as

$$
\begin{aligned}
& \sum_{k_{2}^{\prime}} \tilde{X}_{1,2}\left(1, k_{2}^{\prime}\right) \operatorname{Prob}\left\{m_{1} \text { does not produce, } m_{0} \text { produces } \mid 1, k_{2}^{\prime}\right\} \\
& =\left(1-p_{1}\right) p_{0}\left[\tilde{X}_{1}(1)-\tilde{X}_{1,2}(1,0)\right] \\
& =\left(1-p_{1}\right) p_{0}\left[\tilde{X}_{1}(1)-\tilde{X}_{1}(1) \tilde{X}_{2}(0)\right]+\mathcal{O}(\mu) \\
& =\left(1-p_{1}\right) p_{0}\left[1-\tilde{X}_{2}(0)\right] \tilde{X}_{1}(1)+\mathcal{O}(\mu) .
\end{aligned}
$$

Using Lemma A.1, 


$$
\begin{aligned}
& \sum_{k_{2}^{\prime}} \tilde{X}_{1,2}\left(1, k_{2}^{\prime}\right) \operatorname{Prob}\left\{m_{1} \text { does not produce, } m_{0} \text { produces } \mid 1, k_{2}^{\prime}\right\} \\
& =\left(1-p_{1}\right) \tilde{p}_{0}^{1} \tilde{X}_{1}(1)+\mathcal{O}(\mu) .
\end{aligned}
$$

From (A.10) and (A.14), equation (A.9) can be rewritten as

$$
\tilde{X}_{1}(0)=\left(1-p_{1}+\mathcal{O}(\mu)\right) \tilde{X}_{1}(0)+\left(1-p_{1}\right) \tilde{p}_{0}^{1} \tilde{X}_{1}(1)+\mathcal{O}(\mu) .
$$

Similar arguments are used to obtain equations for $\tilde{X}_{1}\left(k_{1}\right), k_{1}=$ $1, \ldots, N_{1}$. As a result, we have the following:

$$
\begin{aligned}
\tilde{X}_{1}(0)= & \left(1-p_{1}+\mathcal{O}(\mu)\right) \tilde{X}_{1}(0)+\left(1-p_{1}\right) \tilde{p}_{0}^{1} \tilde{X}_{1}(1), \\
\tilde{X}_{1}(1)= & p_{1} \tilde{X}_{1}(0)+\left[p_{1} \tilde{p}_{0}^{1}+\left(1-p_{1}\right)\left(1-\tilde{p}_{0}^{1}\right)+\mathcal{O}(\mu)\right] \tilde{X}_{1}(1) \\
& +\left(1-p_{1}\right) \tilde{p}_{0}^{1} \tilde{X}_{1}(2),
\end{aligned}
$$

$\tilde{X}_{1}\left(k_{1}\right)=p_{1}\left(1-\tilde{p}_{0}^{1}\right) \tilde{X}_{1}\left(k_{1}-1\right)+\left[p_{1} \tilde{p}_{0}^{1}+\left(1-p_{1}\right)\left(1-\tilde{p}_{0}^{1}\right)+\mathcal{O}(\mu)\right] \tilde{X}_{1}\left(k_{1}\right)$

$$
+\left(1-p_{1}\right) \tilde{p}_{0}^{1} X_{1}\left(k_{1}+1\right), k_{1}=2, \ldots, \mathrm{N}_{1}-1,
$$

$\tilde{X}_{1}\left(N_{1}\right)=p_{1}\left(1-\tilde{p}_{0}^{1}\right) \tilde{X}_{1}\left(N_{1}-1\right)+\left[1-\tilde{p}_{0}^{1}+p_{1} \tilde{p}_{0}^{1}+\mathcal{O}(\mu)\right] X_{1}\left(N_{1}\right)$.

This can be written in matrix form as:

$$
\tilde{X}_{1}=(A+\Delta A) \tilde{X}_{1}, \tilde{X}_{1}=\left[\tilde{X}_{1}(0), \ldots, \tilde{X}_{1}\left(N_{1}\right)\right]^{T}
$$

where

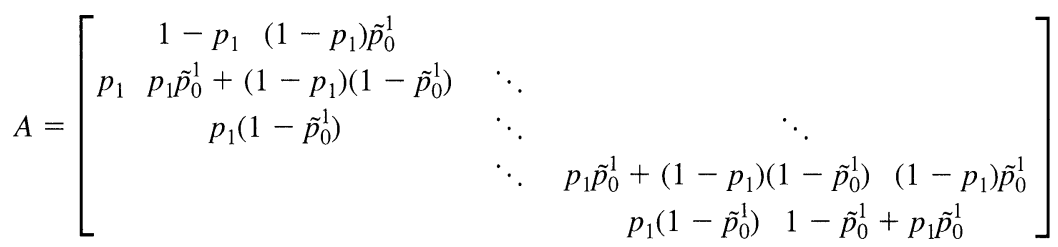


and $\Delta A$ is a diagonal matrix with diagonal elements all of the order $\mathcal{O}(\mu)$, and therefore $\|\Delta A\| \sim \mathcal{O}(\mu)$.

From Lemma A.5 in [1], the stationary probability $\bar{X}_{1}(\cdot)$ of line $L_{1}$ is described by $\bar{X}_{1}=A \bar{X}_{1}$, where $A$ is defined by (A.18). Since $A$ is the state transition matrix of an ergodic Markov chain, $\lambda=1$ is an eigenvalue of multiplicity 1 . Therefore, using the perturbation theory [16],

$$
\tilde{X}_{1}\left(k_{1}\right)=\bar{X}_{1}\left(k_{1}\right)+\mathcal{O}(\mu), \quad k_{1}=0, \ldots, N_{1} .
$$

Analogously, we can prove that

$$
\tilde{X}_{2}\left(k_{2}\right)=\bar{X}_{2}\left(k_{2}\right)+\mathcal{O}(\mu), \quad k_{2}=0, \ldots, N_{2} .
$$

Lemma A.2 shows that if $\bar{X}_{1}(\cdot)$ and $\bar{X}_{2}(\cdot)$ are known, then they can be used to estimate the stationary probability distributions of buffer occupancy $\tilde{X}_{1}(\cdot)$ and $\tilde{X}_{2}(\cdot)$. Our next goal is to estimate $\bar{X}_{1}(0)$ and $\bar{X}_{2}(0)$. Lemma A.3 shows that this can be accomplished using the recursive procedure (3.3).

Lemma A.3: Under Numerical Fact 3.1,

$$
\bar{X}_{i}(0)=X_{i}(0)+\mathcal{O}(\mu), i=1,2,
$$

where $\bar{X}_{1}(0)$ and $\bar{X}_{2}(0)$ are, respectively, stationary probabilities that buffers $b_{1}$ and $b_{2}$ in lines $L_{1}$ and $L_{2}$ are empty, and $X_{1}(0)$ and $X_{2}(0)$ are the steady states of the recursive procedure (3.3).

Proof: Using Lemma A.5 of [1],

$$
\bar{X}_{1}(0)=Q\left(p_{1}, \tilde{p}_{0}^{1}, N_{1}\right)
$$

Since $\tilde{p}_{0}^{1}=p_{0}\left[1-\tilde{X}_{2}(0)\right]+\mathscr{O}(\mu)$ from LemmaA.1,

$$
\bar{X}_{1}(0)=Q\left(p_{1}, p_{0}\left[1-\tilde{X}_{2}(0)\right]+\mathscr{O}(\mu), N_{1}\right) .
$$

From Lemma A.2 and Lemma B.1 of [6], 


$$
\begin{aligned}
\bar{X}_{1}(0) & =Q\left(p_{1}, p_{0}\left[1-\bar{X}_{2}(0)\right]+\mathcal{O}(\mu), N_{1}\right) \\
& =\mathrm{Q}\left(p_{1}, p_{0}\left[1-\overline{\mathrm{X}}_{2}(0)\right], N_{1}\right)+g_{2}\left(p_{1}, p_{0}\left[1-\overline{\mathrm{X}}_{2}(0)\right], N_{1}\right) \mathcal{O}(\mu) \\
& =\mathrm{Q}\left(p_{1}, p_{0}\left[1-\bar{X}_{2}(0)\right], N_{1}\right)+\mathcal{O}(\mu)
\end{aligned}
$$

where

$$
g_{2}(x, y, N)=\left\{\begin{array}{c}
\frac{x-\left[x+N \frac{x(y-x)}{y(1-y)}\right] \alpha^{N}}{y^{2}\left(1-\frac{x}{y} \alpha^{N}\right)^{2}}, x \neq y, \\
\frac{N(N+1-2 x)}{2 x(N+1-x)^{2}}, \quad x=y,
\end{array}\right.
$$

and

$$
\alpha=\frac{x(1-y)}{y(1-x)} .
$$

Similarly,

$$
\bar{X}_{2}(0)=Q\left(p_{2}, p_{0}\left[1-\bar{X}_{1}(0)\right], N_{2}\right)+\mathcal{O}(\mu) .
$$

As it follows from Lemma 3.1, the steady state equations of recursive procedure (3.3) are given below:

$$
\begin{aligned}
& X_{1}(0)=Q\left(p_{1}, p_{0}\left[1-X_{2}(0)\right], N_{1}\right), \\
& X_{2}(0)=Q\left(p_{2}, p_{0}\left[1-X_{1}(0)\right], N_{2}\right) .
\end{aligned}
$$

Equations (A.21) and (A.22) show that $\bar{X}_{1}(0)$ and $\bar{X}_{2}(0)$ solve equations (A.23) and (A.24) with error $O(\mu)$, i.e.,

$$
\bar{X}_{i}(0)=X_{i}(0)+\odot(\mu), i=1,2 .
$$


According to Lemma 3.1, equations (A.23) and (A.24), i.e.,

$$
\begin{aligned}
& X_{1}(0)=Q\left(p_{1}, p_{0}\left[1-X_{2}(0)\right], N_{1}\right), \\
& X_{2}(0)=Q\left(p_{2}, p_{0}\left[1-X_{1}(0)\right], N_{2}\right),
\end{aligned}
$$

have a solution $\left(X_{1}(0), X_{2}(0)\right)$. We show below that this solution is, in fact, unique. In order to prove this, introduce 2 two-machine-one-buffer serial production lines, $L_{i}, i=1,2$, where the first machine of $L_{1}$ has the production rate in isolation $p_{1}$, the second $p_{0}\left[1-X_{2}(0)\right]$, and the buffer capacity $N_{1}$; analogously, the first machine of $L_{2}$ is characterized by $p_{2}$, the second by $p_{0}\left[1-X_{1}(0)\right]$, and the buffer by $N_{2}$. Let $P R_{2}\left(p_{x}, p_{y}, N\right)$ denote the production rate of a two-machine-one-buffer serial line, i.e., according to [1],

$$
P R_{2}\left(p_{x}, p_{y}, N\right)=p_{x}\left[1-Q\left(p_{y}, p_{x}, N\right)\right]=p_{y}\left[1-Q\left(p_{x}, p_{y}, N\right)\right]
$$

Then, the following properties hold:

Lemma A.4: $\quad$ Let $P R_{L_{i}}, i=1,2$, be the production rate of line $L_{i}$. Then

$$
\begin{aligned}
& P R_{L_{1}}=P R_{2}\left(p_{1}, p_{0}\left[1-X_{2}(0)\right], N_{1}\right), \\
& P R_{L_{2}}=P R_{2}\left(p_{2}, p_{0}\left[1-X_{1}(0)\right], N_{2}\right) .
\end{aligned}
$$

In addition,

$$
P R=P R_{L_{1}}=P R_{L_{2}}
$$

Proof: Using Lemma A.5 of [1], we obtain

$$
\begin{aligned}
P R_{L_{1}} & =p_{0}\left[1-X_{2}(0)\right]\left[1-Q\left(p_{1}, p_{0}\left[1-X_{2}(0)\right], N_{1}\right)\right] \\
& =p_{1}\left[1-Q\left(p_{0}\left[1-X_{2}(0)\right], p_{1}, N_{1}\right)\right] \\
& =P R_{2}\left(p_{1}, p_{0}\left[1-X_{2}(0)\right], N_{1}\right),
\end{aligned}
$$




$$
\begin{aligned}
P R_{L_{2}} & =p_{0}\left[1-X_{1}(0)\right]\left[1-Q\left(p_{2}, p_{0}\left[1-X_{1}(0)\right], N_{2}\right)\right] \\
& =p_{2}\left[1-Q\left(p_{0}\left[1-X_{1}(0)\right], p_{2}, N_{2}\right)\right] \\
& =P R_{2}\left(p_{2}, p_{0}\left[1-X_{1}(0)\right], N_{2}\right) .
\end{aligned}
$$

Moreover, using (A.23) and (A.24),

$$
\begin{aligned}
& P R_{L_{1}}=p_{0}\left[1-X_{2}(0)\right]\left[1-X_{1}(0)\right], \\
& P R_{L_{2}}=p_{0}\left[1-X_{1}(0)\right]\left[1-X_{2}(0)\right] .
\end{aligned}
$$

Therefore, from (3.5),

$$
P R=P R_{L_{1}}=P R_{L_{2}}
$$

Lemma A.5: The equilibrium equations (A.23) and (A.24) of recursive procedure (3.3) have a unique solution.

Proof: By contradiction: Assume that along with the solution $\left(X_{1}(0)\right.$, $\left.X_{2}(0)\right)$ to equations (A.23) and (A.24), there exists another solution $\left(\hat{X}_{1}(0)\right.$, $\left.\hat{X}_{2}(0)\right)$. Suppose that $\hat{X}_{1}(0)>X_{1}(0)$. Then, from Lemma A.4,

$$
\begin{aligned}
& \widehat{P R}_{L_{2}}=P R_{2}\left(p_{2}, p_{0}\left[1-\hat{X}_{1}(0)\right], N_{2}\right), \\
& P R_{L_{2}}=P R_{2}\left(p_{2}, p_{0}\left[1-X_{1}(0)\right], N_{2}\right) .
\end{aligned}
$$

Since function $P R_{2}\left(p_{x}, p_{y}, N\right)$ is monotonically increasing in $p_{y}$,

$$
\widehat{P R}_{L_{2}}<P R_{L_{2}}
$$

By Lemma A.4,

$$
\begin{aligned}
\widehat{P R_{L_{2}}}={\widehat{P R_{L_{1}}}} & =P R_{2}\left(p_{1}, p_{0}\left[1-\hat{X}_{2}(0)\right], N_{1}\right) \\
& <P R_{L_{2}}=P R_{L_{1}}=P R_{2}\left(p_{1}, p_{0}\left[1-X_{2}(0)\right], N_{1}\right)
\end{aligned}
$$


Again, using the monotonically increasing property of function $P R_{2}\left(p_{x}, p_{y}\right.$, $N)$ in $p_{y}$,

$$
p_{0}\left[1-\hat{X}_{2}(0)\right]<p_{0}\left[1-X_{2}(0)\right]
$$

Since function $Q(x, y, N)$ is a monotonically increasing function in $y$,

$$
Q\left(p_{1}, p_{0}\left[1-\hat{X}_{2}(0)\right], N_{1}\right)<Q\left(p_{1}, p_{0}\left[1-X_{2}(0)\right], N_{1}\right),
$$

i.e.,

$$
\hat{X}_{1}(0)<X_{1}(0)
$$

which is a contradiction. Hence,

$$
\hat{X}_{1}(0) \leq X_{1}(0)
$$

Assuming that $\hat{X}_{1}(0)<X_{1}(0)$, and proceeding analogously, yields $\hat{X}_{1}(0)$ $\geq X_{1}(0)$. Therefore, we conclude that

$$
\hat{X}_{1}(0)=X_{1}(0)
$$

Similar arguments are used to prove that $\hat{X}_{2}(0)=X_{2}(0)$. Therefore, the solution $\left(X_{1}(0), X_{2}(0)\right)$ is unique.

Proof of Theorem 3.1: According to Lemma A.2,

$$
\tilde{X}_{i}(0)=\bar{X}_{i}(0)+\mathcal{O}(\mu), \quad i=1,2
$$

According to Lemmas A.3 and A.5,

$$
\bar{X}_{i}(0)=X_{i}(0)+\mathcal{O}(\mu), \quad i=1,2 .
$$

Therefore,

$$
\tilde{X}_{i}(0)=X_{i}(0)+\mathcal{O}(\mu), i=1,2 .
$$


Proof of Corollary 3.2: Using Lemma A.2,

$$
\tilde{X}_{1}\left(k_{1}\right)=\bar{X}_{1}\left(k_{1}\right)+\mathcal{O}(\mu), k_{1}=0, \ldots, N_{1}
$$

By Lemma A.5 of [1] and Lemma A.2,

$$
\bar{X}_{1}\left(k_{1}\right)=\bar{X}_{1}(0)\left(\frac{1}{1-\tilde{p}_{0}^{1}}\right) \tilde{\alpha}_{1}^{k_{1}}, k_{1}=1, \ldots, N_{1},
$$

where $\tilde{\alpha}_{1}=\frac{p_{1}\left(1-\tilde{p}_{0}^{1}\right)}{\tilde{p}_{0}^{1}\left(1-p_{1}\right)}$ and $\tilde{p}_{0}^{1}=p_{0}\left[1-\tilde{X}_{2}(0)\right]+\mathcal{O}(\mu)($ Lemma A.1). Using Lemma A.3 and Theorem 3.1, equation (A.26) can be rewritten as

$$
\bar{X}\left(k_{1}\right)=X_{1}(0)\left(\frac{1}{1-p_{0}\left[1-X_{2}(0)\right]}\right) \alpha_{1}^{k_{1}}+\mathcal{O}(\mu), k_{1}=1, \ldots, N_{1},
$$

where $\alpha_{1}=\frac{p_{1}\left(1-p_{0}\left[1-X_{2}(0)\right]\right)}{p_{0}\left[1-X_{2}(0)\right]\left(1-p_{1}\right)}$. From (A.25) and (A.27),

$$
\tilde{X}_{1}\left(k_{1}\right)=X_{1}(0)\left(\frac{1}{1-p_{0}\left[1-X_{2}(0)\right]}\right) \alpha_{1}^{k_{1}}+\sigma(\mu), k_{1}=1, \ldots, N_{1} \text {. }
$$

Similar arguments are used to evaluate $\tilde{X}_{2}\left(k_{2}\right), k_{2}=1, \ldots, N_{2}$. As a result,

$$
\tilde{X}_{2}\left(k_{2}\right)=X_{2}(0)\left(\frac{1}{1-p_{0}\left[1-X_{1}(0)\right]}\right) \alpha_{2}^{k_{2}}+\sigma(\mu), k_{2}=1, \ldots, N_{2},
$$

where $\alpha_{2}=\frac{p_{2}\left(1-p_{0}\left[1-X_{1}(0)\right]\right)}{p_{0}\left[1-X_{1}(0)\right]\left(1-p_{2}\right)}$. Therefore, from (3.6), we conclude that

$$
\tilde{X}_{i}\left(k_{i}\right)=X_{i}\left(k_{i}\right)+\mathcal{O}(\mu), k_{i}=1, \ldots, N_{i}, i=1,2 .
$$


Proof of Corollary 3.3: Let $P R_{2}\left(p_{x}, p_{y}, N\right)$ denote the production rate of a two-machine-one-buffer serial line [1]. Then, we have the following two properties:

$(\alpha)$. Function $P R_{2}\left(p_{x}, p_{y}, N\right)$ is monotonically increasing in $p_{x}, p_{y}$, and $N$ (Lemma A.5 of [1]).

(B). Function $Q(x, y, N)$, is monotonically decreasing in $x$ and $N$, and monotonically increasing in $y$ (Lemma A.1 of [1]).

Consider two assembly systems (i)-(vi), the first of which is described by parameters $p_{i}, i=0,1,2$, and $N_{i}, i=1,2$, and the second by parameters $\hat{p}_{i} \geq p_{i}, i=0,1,2$, and $\hat{N}_{i} \geq N_{i}, i=1,2$. Let $X_{i}(0)$ and $\hat{X}_{i}(0), i=$ 1,2 , denote the steady state of recursive procedure (3.3) for the first and second assembly systems, respectively. We prove Corollary 3.3 by contradiction.

Assume

$$
P R\left(p_{1}, p_{2}, p_{0}, N_{1}, N_{2}\right)>P R\left(\hat{p}_{1}, \hat{p}_{2}, \hat{p}_{0}, \hat{N}_{1}, \hat{N}_{2}\right)
$$

Thus,

$$
\begin{aligned}
P R_{2}\left(p_{1}, p_{0}\left[1-X_{2}(0)\right], N_{1}\right) & =P R\left(p_{1}, p_{2}, p_{0}, N_{1}, N_{2}\right) \\
& >P R\left(\hat{p}_{1}, \hat{p}_{2}, \hat{p}_{0}, \hat{N}_{1}, \hat{N}_{2}\right) \\
& =P R_{2}\left(\hat{p}_{1}, \hat{p}_{0}\left[1-\hat{X}_{2}(0)\right], \hat{N}_{1}\right)
\end{aligned}
$$

Then, by $(\alpha)$,

$$
p_{0}\left[1-X_{2}(0)\right]>\hat{p}_{0}\left[1-\hat{X}_{2}(0)\right]
$$

Hence,

$$
\hat{X}_{2}(0)>X_{2}(0)
$$

Since $\hat{X}_{2}(0)=Q\left(\hat{p}_{2}, \hat{p}_{0}\left[1-\hat{X}_{1}(0)\right], \hat{N}_{2}\right)$ and $X_{2}(0)=Q\left(p_{2}, p_{0}\left[1-X_{1}(0)\right]\right.$, $N_{2}$ ) and $(\beta)$, 


$$
\hat{p}_{0}\left[1-\hat{X}_{1}(0)\right]>p_{0}\left[1-X_{1}(0)\right]
$$

Again, using $(\alpha)$,

$$
P R_{2}\left(\hat{p}_{2}, \hat{p}_{0}\left[1-\hat{X}_{1}(0)\right], \hat{N}_{2}\right)>P R_{2}\left(p_{2}, p_{0}\left[1-X_{1}(0)\right], N_{2}\right),
$$

i.e.,

$$
P R\left(p_{1}, p_{2}, p_{0}, N_{1}, N_{2}\right)<P R\left(\hat{p}_{1}, \hat{p}_{2}, \hat{p}_{0}, \hat{N}_{1}, \hat{N}_{2}\right)
$$

which contradicts the assumption. Therefore, we conclude that $\operatorname{PR}\left(p_{1}, p_{2}\right.$, $p_{0}, N_{1}, N_{2}$ ) is a monotonically increasing function of its arguments.

\section{APPENDIX B. PROOFS FOR SECTION 4}

To prove Theorem 4.1, Corollary 4.1 and Theorem 4.2, we need Lemmas B.1-B.5. To formulate and prove them, we recall a few results from [1]:

Define function $f_{N}(x, y)$ as follows:

$$
f_{N}(x, y)=[1-Q(x, y, N)][1-Q(y, x, N)]
$$

where $Q(x, y, N)$ is given in (3.3). Then

$(\alpha)$. function $f_{N}(x, y)$ can be represented as

$$
f_{N}(x, y)=\left[\frac{g\left(1-\alpha^{N}\right)}{1-\alpha^{N} g^{2}}\right]^{2},
$$

where

$$
\begin{aligned}
& g=\sqrt{\frac{x}{y}}, \\
& \alpha=\frac{x(1-y)}{y(1-x)},
\end{aligned}
$$

(Lemma B.1 of [1]); 
( $\beta)$. under the constraint $x y=p=$ const, function $f_{N}(x, y)$, achieves its maximum value if and only if $x=y$. Furthermore,

$$
f_{N}(\sqrt{p}, \sqrt{p})=\left[\frac{N}{N+1-\sqrt{p}}\right]^{2}
$$

(Lemma B.2 of [1]).

Lemma B.1: Let $c_{1}=\sqrt{p_{1} p_{0}\left[1-X_{2}(0)\right]}$ and $c_{2}=\sqrt{p_{1} p_{0}\left[1-X_{1}(0)\right]}$. Then

$$
c_{i} \geq P R\left(\frac{N_{i}+1}{N_{i}+P R}\right), i=1,2 .
$$

The equality takes place if and only if $p_{1}=p_{0}\left[1-X_{2}(0)\right]$ and $p_{2}=$ $p_{0}\left[1-X_{1}(0)\right]$.

Proof: From Lemma A.4,

$$
\begin{aligned}
c_{1}^{2} & =p_{1} p_{0}\left[1-X_{2}(0)\right] \\
& =\frac{p_{1}\left[1-Q\left(p_{0}\left[1-X_{2}(0)\right], p_{1}, N_{1}\right)\right] p_{0}\left[1-X_{2}(0)\right]\left[1-Q\left(p_{1}, p_{0}\left[1-X_{2}(0)\right], N_{1}\right)\right]}{\left[1-Q\left(p_{0}\left[1-X_{2}(0)\right], p_{1}, N_{1}\right)\right]\left[1-Q\left(p_{1}, p_{0}\left[1-X_{2}(0)\right], N_{1}\right)\right]} \\
& =\frac{P R^{2}}{f_{N_{1}}\left(p_{1}, p_{0}\left[1-X_{2}(0)\right]\right)} .
\end{aligned}
$$

By $(\beta)$ and the definition of $c_{1}$,

$$
f_{N_{1}}\left(p_{1}, p_{0}\left[1-X_{2}(0)\right]\right) \leq\left[\frac{N_{1}}{N_{1}+1-c_{1}}\right]^{2}
$$

with the equality taking place if and only if $p_{1}=p_{0}\left[1-X_{2}(0)\right]$, so

$$
c_{1}^{2} \geq \frac{P R^{2}}{\left[\frac{N_{1}}{N_{1}+1-c_{1}}\right]^{2}},
$$




$$
\begin{aligned}
c_{1} & \geq \frac{P R\left(N_{1}+1-c_{1}\right)}{N_{1}}, \\
c_{1}\left(1+\frac{P R}{N_{1}}\right) & \geq \frac{P R\left(N_{1}+1\right)}{N_{1}}, \\
c_{1} & \geq \frac{P R\left(N_{1}+1\right)}{N_{1}+P R} .
\end{aligned}
$$

Analogously,

$$
c_{2} \geq \frac{P R\left(N_{2}+1\right)}{N_{2}+P R} .
$$

LEMMA B.2: The total workforce, $p^{*}$, necessary to achieve the production rate value $P R$, is bounded by

$$
p^{*} \geq\left(\frac{N_{1}+1}{N_{1}+P R}\right)^{2}\left(\frac{N_{2}+1}{N_{2}+P R}\right)^{2} P R^{3} .
$$

The equality takes place if and only if

$$
\begin{aligned}
& p_{1}=p_{0}\left[1-X_{2}(0)\right], \\
& p_{2}=p_{0}\left[1-X_{1}(0)\right] .
\end{aligned}
$$

Proof: From (3.5) and Lemma A.4,

$$
\begin{aligned}
P R & =p_{0}\left[1-X_{1}(0)\right]\left[1-X_{2}(0)\right] \\
& =\frac{p_{1} p_{0}\left[1-X_{2}(0)\right] p_{2} p_{0}\left[1-X_{1}(0)\right]}{p_{1} p_{2} p_{0}} \\
& =\frac{c_{1}^{2} c_{2}^{2}}{p_{1} p_{2} p_{0}} .
\end{aligned}
$$

Thus, 


$$
p_{1} p_{2} p_{0}=p^{*}=\frac{c_{1}^{2} c_{2}^{2}}{P R}
$$

By Lemma B.1,

$$
p^{*} \geq\left(\frac{N_{1}+1}{N_{1}+P R}\right)^{2}\left(\frac{N_{2}+1}{N_{2}+P R}\right)^{2} P R^{3}
$$

with the equality taking place if and only if

$$
\begin{aligned}
& p_{1}=p_{0}\left[1-X_{2}(0)\right], \\
& p_{2}=p_{0}\left[1-X_{1}(0)\right] .
\end{aligned}
$$

Lemma B. 2 gives a lower bound on the workforce necessary to accomplish a desired production rate. The following lemma shows that this bound is achievable.

LeMmA B.3: The conditions $p_{1}=p_{0}\left[1-X_{2}(0)\right]$ and $p_{2}=p_{0}\left[1-X_{1}(0)\right]$ are achieved if and only if the workforce is distributed as follows:

$$
\begin{aligned}
& p_{1}=\left(\frac{N_{1}+1}{N_{1}+P R}\right) P R, \\
& p_{2}=\left(\frac{N_{2}+1}{N_{2}+P R}\right) P R, \\
& p_{0}=\left(\frac{N_{1}+1}{N_{1}+P R}\right)\left(\frac{N_{2}+1}{N_{2}+P R}\right) P R .
\end{aligned}
$$

Proof: $\quad$ Suppose $p_{1}=p_{0}\left[1-X_{2}(0)\right]$ and $p_{2}=p_{0}\left[1-X_{1}(0)\right]$. Then, from Lemma A.4, 


$$
\begin{aligned}
P R & =p_{1}\left[1-Q\left(p_{0}\left[1-X_{2}(0)\right], p_{1}, N_{1}\right)\right] \\
& =p_{1}\left(1-\frac{1-p_{1}}{N_{1}+1-p_{1}}\right) \\
& =\frac{p_{1} N_{1}}{N_{1}+1-p_{1}}
\end{aligned}
$$

Solving (B.10) for $p_{1}$, we obtain

$$
p_{1}=p_{0}\left[1-X_{2}(0)\right]=\left(\frac{N_{1}+1}{N_{1}+P R}\right) P R
$$

Similarly, we have

$$
p_{2}=p_{0}\left[1-X_{1}(0)\right]=\left(\frac{N_{2}+1}{N_{2}+P R}\right) P R .
$$

Using Lemma A.4 and recalling that $p_{1}=p_{0}\left[1-X_{2}(0)\right]$ and $p_{2}=p_{0}[1-$ $\left.X_{1}(0)\right]$,

$$
\begin{aligned}
P R & =p_{0}\left[1-X_{1}(0)\right]\left[1-X_{2}(0)\right] \\
& =p_{0}\left[1-Q\left(p_{1}, p_{0}\left[1-X_{2}(0)\right], N_{1}\right)\right]\left[1-Q\left(p_{2}, p_{0}\left[1-X_{1}(0)\right], N_{2}\right)\right] \\
& =p_{0}\left(1-\frac{1-p_{1}}{N_{1}+1-p_{1}}\right)\left(1-\frac{1-p_{2}}{N_{2}+1-p_{2}}\right) \\
& =p_{0}\left(\frac{N_{1}}{N_{1}+1-p_{1}}\right)\left(\frac{N_{2}}{N_{2}+1-p_{2}}\right) .
\end{aligned}
$$

Substitute for $p_{1}$ using (B.11) and for $p_{2}$ using (B.12). Then, 


$$
\begin{aligned}
P R & =p_{0}\left(\frac{N_{1}}{N_{1}+1-\left(\frac{N_{1}+1}{N_{1}+P R}\right) P R}\right)\left(\frac{N_{2}}{N_{2}+1-\left(\frac{N_{2}+1}{N_{2}+P R}\right) P R}\right) \\
& =p_{0}\left(\frac{N_{1}+P R}{N_{1}+1}\right)\left(\frac{N_{2}+P R}{N_{2}+1}\right)
\end{aligned}
$$

Therefore,

$$
p_{0}=\left(\frac{N_{1}+1}{N_{1}+P R}\right)\left(\frac{N_{2}+1}{N_{2}+P R}\right) P R
$$

Now suppose that the workforce is distributed as in (B.9). Next we show that this implies that $p_{1}=p_{0}\left[1-X_{2}(0)\right]$ and $p_{2}=p_{0}\left[1-X_{1}(0)\right]$. By Lemma A.5, there is a unique solution to (A.23) and (A.24). From (B.11) and (B.12), we claim that this solution is

$$
\begin{aligned}
& X_{1}(0)=1-\left(\frac{N_{2}+1}{N_{2}+P R}\right) \frac{P R}{p_{0}}=\frac{1-P R}{N_{1}+1} \\
& X_{2}(0)=1-\left(\frac{N_{1}+1}{N_{1}+P R}\right) \frac{P R}{p_{0}}=\frac{1-P R}{N_{2}+1} .
\end{aligned}
$$

Due to the uniqueness of this solution, we only need to prove that (B.15) is indeed a solution. Consider

$$
\begin{aligned}
Q\left(p_{1}, p_{0}\left[1-X_{2}(0)\right], N_{1}\right)= & Q\left(\frac{N_{1}+1}{N_{1}+P R} P R, \frac{N_{1}+1}{N_{1}+P R} P R, N_{1}\right) \\
= & \frac{1-\frac{N_{1}+1}{N_{1}+P R} P R}{N_{1}+1-\frac{N_{1}+1}{N_{1}+P R} P R}
\end{aligned}
$$




$$
\begin{aligned}
& =\frac{1-\mathrm{PR}}{\mathrm{N}_{1}+1} \\
& =X_{1}(0) .
\end{aligned}
$$

Similarly,

$$
\begin{aligned}
Q\left(p_{2}, p_{0}\left[1-X_{1}(0)\right], N_{2}\right) & =Q\left(\frac{N_{2}+1}{N_{2}+P R} P R, \frac{N_{2}+1}{N_{2}+P R} P R, N_{2}\right) \\
& =\frac{1-\frac{N_{2}+1}{N_{2}+P R} P R}{N_{2}+1-\frac{N_{2}+1}{N_{2}+P R} P R} \\
& =\frac{1-P R}{N_{2}+1} \\
& =X_{2}(0) .
\end{aligned}
$$

Therefore, equations (B.16) and (B.17) imply that

$$
\begin{aligned}
& p_{1}=p_{0}\left[1-X_{2}(0)\right], \\
& p_{2}=p_{0}\left[1-X_{1}(0)\right] .
\end{aligned}
$$

LEMMA B.4: The minimum workforce, $p_{\min }^{*}$, required to achieve production rate $P R$ is given by

$$
p_{\text {min }}^{*}=\left(\frac{N_{1}+1}{N_{1}+P R}\right)^{2}\left(\frac{N_{2}+1}{N_{2}+P R}\right)^{2} P R^{3} .
$$

In addition, this production rate is achieved if and only if $p^{*}$ is distributed among $p_{1}, p_{2}$ and $p_{0}$ so that $p_{1} p_{2} p_{0}=p^{*}, p_{1}=p_{0}\left[1-X_{2}(0)\right]$ and $p_{2}=$ $p_{0}\left[1-X_{1}(0)\right]$.

Proof: According to Lemma B.2, 


$$
p_{\text {min }}^{*} \geq\left(\frac{N_{1}+1}{N_{1}+P R}\right)^{2}\left(\frac{N_{2}+1}{N_{2}+P R}\right)^{2} P R^{3},
$$

and equality is achieved if and only if $p_{1}=p_{0}\left[1-X_{2}(0)\right]$ and $p_{2}=p_{0}$ [1 $\left.-X_{1}(0)\right]$. By Lemma B.3, this lower bound is obtained with the workforce distribution as specified in equation (B.9).

LEMMA B.5: The minimum workforce, $p_{\min }^{*}$, necessary to obtain the production rate $P R$ is a monotonically increasing function of $P R$.

Proof: By Lemma B. $4, p_{\min }^{*}$ is given by

$$
p_{\text {min }}^{*}=\left(\frac{N_{1}+1}{N_{1}+P R}\right)^{2}\left(\frac{N_{2}+1}{N_{2}+P R}\right)^{2} P R^{3} .
$$

Differentiation of $p_{\min }^{*}$ with respect to $P R$ yields

$$
\begin{aligned}
\frac{\partial p_{\min }^{*}}{\partial P R}= & -2\left(\frac{N_{1}+1}{N_{1}+P R}\right) \frac{\left(N_{1}+1\right)}{\left(N_{1}+P R\right)^{2}}\left(\frac{N_{1}+1}{N_{1}+P R}\right)^{2} P R^{3} \\
& -\left(\frac{N_{1}+1}{N_{1}+P R}\right)^{2} 2\left(\frac{N_{2}+1}{N_{2}+P R}\right) \frac{\left(N_{2}+1\right)}{\left(N_{2}+P R\right)^{2}} P R^{3} \\
& +\left(\frac{N_{1}+1}{N_{1}+P R}\right)^{2}\left(\frac{N_{1}+1}{N_{1}+P R}\right)^{2} 3 P R^{2} \\
& =\frac{\left(N_{1}+1\right)^{2}\left(N_{1}+P R\right)\left(N_{2}+1\right)^{2}\left(N_{2}+P R\right) P R^{2}\left[3 N_{1} N_{2}+P R\left(N_{1}+N_{2}-P R\right)\right]}{\left(N_{1}+P R\right)^{4}\left(N_{2}+P R\right)^{4}}>0 .
\end{aligned}
$$

Therefore, $p_{\min }^{*}$ is monotonically increasing in $P R$.

Proof of Theorem 4.1: "Only if": Suppose the assembly system is unimprovable, but either $p_{1} \neq p_{0}\left[1-X_{2}(0)\right]$ or $p_{2} \neq p_{0}\left[1-X_{1}(0)\right]$. Then by Lemma B.4, $p^{*}>p_{\text {min }}^{*}$. Thus, from Lemma B.5, workforce $p^{*}$ optimally distributed can achieve a larger production rate, which is a contradiction. 
The "if" part follows from Lemma B.4. Indeed, if $p_{1}=p_{0}\left[1-X_{2}(0)\right]$ and $p_{2}=p_{0}\left[1-X_{1}(0)\right]$, the assembly system is unimprovable.

Proof of Corollary 4.1: From Theorem 3.1 and Corollary 3.2, the distributions of buffer occupancy $\widetilde{X}_{1}(\cdot)$ and $\widetilde{\mathrm{X}}_{2}(\cdot)$ can be approximated with error $\mathcal{O}(\mu)$ by $X_{1}(\cdot)$ and $X_{2}(\cdot)$. Hence, under Numerical Fact 3.1,

$$
\begin{aligned}
\widetilde{c b}_{1} & =\operatorname{Prob}\left\{b_{1} \text { is full and } m_{0} \text { fails to take parts from } b_{1}\right\} \\
& =\tilde{X}_{1}\left(N_{1}\right)\left(1-p_{0}\right)+\tilde{X}_{1,2}\left(N_{1}, 0\right) p_{0} \\
& =\tilde{X}_{1}\left(N_{1}\right)\left(1-p_{0}\right)+\tilde{X}_{1}\left(N_{1}\right) \tilde{X}_{2}(0) p_{0}+O(\mu) \\
& =\tilde{X}_{1}\left(N_{1}\right)\left(1-p_{0}\left[1-\tilde{X}_{2}(0)\right]\right)+O(\mu) \\
& =X_{1}\left(N_{1}\right)\left(1-p_{0}\left[1-X_{2}(0)\right]\right)+O(\mu), \\
\widetilde{c s} & =\operatorname{Prob}\left\{b_{1}\right. \text { is empty } \\
& =\tilde{X}_{1}(0) \\
& =\mathrm{X}_{1}(0)+O(\mu) .
\end{aligned}
$$

Using Lemma A.5 of [1],

$$
\begin{aligned}
& \widetilde{c b}_{1}=Q\left(p_{0}\left[1-X_{2}(0)\right], p_{1}, N_{1}\right)+\mathcal{O}(\mu), \\
& \widetilde{c s}_{1}=Q\left(p_{1}, p_{0}\left[1-X_{2}(0)\right], N_{1}\right)+\sigma(\mu) .
\end{aligned}
$$

The expressions for $\widetilde{c b}_{2}$ and $\widetilde{c s}_{2}$ can be derived analogously:

$$
\begin{aligned}
& \widetilde{c b}_{2}=Q\left(p_{0}\left[1-X_{1}(0)\right], p_{2}, N_{2}\right)+\mathcal{O}(\mu), \\
& \widetilde{c s}_{2}=Q\left(p_{2}, p_{0}\left[1-X_{1}(0)\right], N_{2}\right)+\mathcal{O}(\mu) .
\end{aligned}
$$


Since $p_{1}=p_{0}\left[1-X_{2}(0)\right]$ and $p_{2}=p_{0}\left[1-X_{1}(0)\right]$, from (B.20), (B.21), (B.22) and (B.23), the result of statement (a) follows.

Since $p_{1}=p_{0}\left[1-X_{2}(0)\right]$ and $p_{2}=p_{0}\left[1-X_{1}(0)\right]$, from (3.6)

$$
X_{i}\left(k_{i}\right)=\frac{X_{i}(0)}{1-p_{i}}, \quad k_{i}=1, \ldots, N_{i}, \quad i=1,2,
$$

where

$$
X_{i}(0)=\frac{1-p_{i}}{N_{i}+1-p_{i}} .
$$

Thus,

$$
\begin{aligned}
\tilde{\tilde{h}}_{i}=E\left[\tilde{h}_{i}\right] & =\sum_{k_{i}=0}^{N_{i}} k_{i} X_{i}\left(k_{i}\right)+\mathcal{O}(\mu) \\
& =\sum_{k_{i}=1}^{N_{i}} k_{i}\left(\frac{1}{1-p_{i}}\right)\left(\frac{1-p_{i}}{N_{i}+1-p_{i}}\right)+\mathcal{O}(\mu) \\
& =\frac{N_{i}\left(N_{i}+1\right)}{2\left(N_{i}+1-p_{i}\right)}+\mathcal{O}(\mu), i=1,2 .
\end{aligned}
$$

This proves statement (b) of the corollary.

Proof of Theorem 4.2: It has been shown in Lemma B.3 that (4.1) is satisfied if and only if

$$
\begin{aligned}
& p_{1}=\left(\frac{N_{1}+1}{N_{1}+P R^{*}}\right) P R^{*}, \\
& p_{2}=\left(\frac{N_{2}+1}{N_{2}+P R^{*}}\right) P R^{*}, \\
& p_{0}=\left(\frac{N_{1}+1}{N_{1}+P R^{*}}\right)\left(\frac{N_{2}+1}{N_{2}+P R^{*}}\right) P R^{*},
\end{aligned}
$$


where $P R^{*}$ is defined by (4.2). Thus, for an assembly system satisfying (4.1),

$$
p^{*}=p_{1} p_{2} p_{0}=\left(P R^{*}\right)^{3}\left(\frac{N_{1}+1}{N_{1}+P R^{*}}\right)^{2}\left(\frac{N_{2}+1}{N_{2}+P R^{*}}\right)^{2} .
$$

This can be rearranged as

$$
P R^{*}=\left(p^{*}\right)^{\frac{1}{3}}\left(\frac{N_{1}+P R^{*}}{N_{1}+1}\right)^{\frac{2}{3}}\left(\frac{N_{2}+P R^{*}}{N_{2}+1}\right)^{\frac{2}{3}} .
$$

Define a function $f(\cdot)$ as

$$
f(x)=\left(p^{*}\right)^{\frac{1}{3}}\left(\frac{N_{1}+x}{N_{1}+1}\right)^{\frac{2}{3}}\left(\frac{N_{2}+x}{N_{2}+1}\right)^{\frac{2}{3}},
$$

and observe that

$$
P R^{*}=f\left(P R^{*}\right)
$$

We next prove that $x(s+1)=f(x(s))$ is a contraction on $[0,1]$ and, therefore, (B.28) has a unique solution which recursive procedure (4.3) converges to. Calculate

$$
\begin{aligned}
& \frac{d f(x)}{d x}=\left(p^{*}\right)^{\frac{1}{3}} \frac{2}{3}\left(\frac{N_{1}+x}{N_{1}+1}\right)^{-\frac{1}{3}}\left(\frac{1}{N_{1}+1}\right)\left(\frac{N_{2}+x}{N_{2}+1}\right)^{\frac{2}{3}} \\
& +\left(p^{*}\right)^{\frac{1}{3}}\left(\frac{N_{1}+x}{N_{1}+1}\right)^{\frac{2}{3}} \frac{2}{3}\left(\frac{N_{2}+x}{N_{2}+1}\right)^{-\frac{1}{3}}\left(\frac{1}{N_{2}+1}\right) \\
& =\left(p^{*}\right)^{\frac{1}{3}}\left(\frac{N_{1}+x}{N_{1}+1}\right)^{\frac{2}{3}}\left(\frac{N_{2}+x}{N_{2}+1}\right)^{\frac{2}{3}} \frac{2}{3}\left(\frac{1}{N_{1}+x}+\frac{1}{N_{2}+x}\right) .
\end{aligned}
$$


Since $p^{*}<1$ and $\frac{N_{i}+x}{N_{i}+1} \leq 1$ for $0 \leq x \leq 1$, using the assumption $\frac{1}{N_{1}}+$ $\frac{1}{N_{2}} \leq \frac{3}{2}$, we have

$$
\left|\frac{d f(x)}{d x}\right|<1,0 \leq x \leq 1
$$

By the Mean Value Theorem, $\exists c \in[x, y]$ such that

$$
f(x)-f(y)=\left.\frac{d f(x)}{d x}\right|_{x=c}(x-y)
$$

Therefore,

$$
|f(x)-f(y)|<|x-y|,
$$

i.e., $x(s+1)=f(x(s))$ is a contraction on $[0,1]$. This completes the proof of Theorem 4.2.

\section{APPENDIX C. PROOFS FOR SECTION 5}

Proof of Theorem 5.1: Define

$$
f\left(N_{1}, N_{2}, x\right)=\left(p^{*}\right)^{\frac{1}{3}}\left(\frac{N_{1}+x}{N_{1}+1}\right)^{\frac{2}{3}}\left(\frac{N_{2}+x}{N_{2}+1}\right)^{\frac{2}{3}}
$$

and

$$
f^{*}(x)=\max _{\substack{N_{1}, N_{2} \\ N_{1}+N_{2}=N}} f\left(N_{1}, N_{2}, x\right) .
$$

Using the Lagrange multiplier technique, the values $N_{1}^{*}$ and $N_{2}^{*}$ which solve (C.2) can be determined as follows: Introduce

$$
F\left(N_{1}, N_{2}, \lambda\right)=f\left(N_{1}, N_{2}, x\right)+\lambda\left(N_{1}+N_{2}-N^{*}\right) .
$$

Then the optimality conditions, 


$$
\begin{aligned}
& \frac{\partial F\left(N_{1}, N_{2}, \lambda\right)}{\partial N_{1}}=\left(p^{*}\right)^{\frac{1}{3}} \frac{2}{3}\left(\frac{N_{1}+x}{N_{1}+1}\right)^{-\frac{1}{3}} \frac{(1-x)}{\left(N_{1}+1\right)^{2}}\left(\frac{N_{2}+x}{N_{2}+1}\right)^{\frac{2}{3}}+\lambda=0, \\
& \frac{\partial F\left(N_{1}, N_{2}, \lambda\right)}{\partial N_{2}}=\left(p^{*}\right)^{\frac{1}{3}}\left(\frac{N_{1}+x}{N_{1}+1}\right)^{\frac{2}{3}} \frac{2}{3}\left(\frac{N_{2}+x}{N_{2}+1}\right)^{-\frac{1}{3}} \frac{(1-x)}{\left(N_{2}+1\right)^{2}}+\lambda=0,
\end{aligned}
$$

are satisfied if and only if $N_{1}=N_{2}$. Therefore, the solution of (C.2) is given by

$$
N_{i}^{*}=\frac{N^{*}}{2}, i=1,2
$$

Consider now recursive procedure (4.3),

$$
x(s+1)=f\left(N_{1}, N_{2}, x(s)\right),
$$

and recall that, by Theorem 4.2, $\lim _{s \rightarrow x} x(s)=P R\left(p_{1}^{*}, p_{2}^{*}, p_{0}^{*}, N_{1}, N_{2}\right)$, where $p_{i}^{*}, i=0,1,2$, are given by (4.4). Consider now recursive procedure (4.3) defined on the optimal pair $\left(N_{1}^{*}, N_{2}^{*}\right)$ and any other one $\left(N_{1}^{\#}, N_{2}^{*}\right)$ such that $N_{1}^{\#}+N_{2}^{\#}=N^{*}$ :

$$
\begin{aligned}
& x^{*}(s+1)=f\left(N_{1}^{*}, N_{2}^{*}, x^{*}(s)\right), \\
& x^{\#}(s+1)=f\left(N_{1}^{\#}, N_{2}^{\#}, x^{\#}(s)\right) .
\end{aligned}
$$

Assume that the initial conditions for (C.4) and (C.5) are the same:

$$
x^{*}(0)=x^{\#}(0) \in[0,1]
$$

We claim that

$(\alpha) . x^{*}(s) \geq x^{\#}(s), \forall s>0$, i.e.,

$$
P R\left(p_{1}^{*}, p_{2}^{*}, p_{0}^{*}, N_{1}^{*}, N_{2}^{*}\right) \geq P R\left(p_{1}^{*}, p_{2}^{*}, p_{0}^{*}, N_{1}^{*}, N_{2}^{*}\right)
$$

(ß). $X_{1}(0)=X_{2}(0)$.

To prove $(\alpha)$, we use induction. For $s=1$, 


$$
x^{*}(1) \geq x^{\#}(1),
$$

because the sequence $N_{1}^{*}$ and $N_{2}^{*}$ solves (C.2). Assume that

$$
x^{*}(s) \geq x^{\sharp}(s), s>1 .
$$

Since

$$
\frac{d f(x)}{d x}=\left(p^{*}\right)^{\frac{1}{3}}\left(\frac{N_{1}+x}{N_{1}+1}\right)^{\frac{2}{3}}\left(\frac{N_{2}+x}{N_{2}+1}\right)^{\frac{2}{3}} \frac{2}{3}\left(\frac{1}{N_{1}+x}+\frac{1}{N_{2}+x}\right)>0,
$$

i.e., $f\left(N_{1}, N_{2}, x\right)$ is monotonically increasing in $x$, and since the sequence $N_{1}^{*}$ and $N_{2}^{*}$ solves (C.2),

$$
\begin{aligned}
x^{*}(s+1) & =f\left(N_{1}^{*}, N_{2}^{*}, x^{*}(s)\right) \\
& \geq f\left(N_{1}^{*}, N_{2}^{*}, x^{\#}(s)\right) \\
& \geq f\left(\mathrm{~N}_{1}^{\#}, \mathrm{~N}_{2}^{\#}, x^{\#}(s)\right) \\
& =x^{\#}(s+1), \forall s .
\end{aligned}
$$

For statement ( $\beta$ ), from (C.2) and (B.15),

$$
\begin{aligned}
& X_{1}(0)=\frac{1-P R}{N_{1}+1}=\frac{1-P R}{\frac{N^{*}}{2}+1}, \\
& X_{2}(0)=\frac{1-P R}{N_{2}+1}=\frac{1-P R}{\frac{N^{*}}{2}+1} .
\end{aligned}
$$

Proof of Corollary 5.1: By conditions (4.1) and (5.1),

$$
p_{1}=p_{2}=p_{0}\left[1-X_{1}(0)\right]
$$


Substituting (C.3) into (B.25), we have

$$
\overline{\tilde{h}}_{i}=\frac{\frac{N^{*}}{2}\left(\frac{N^{*}}{2}+1\right)}{2\left(\frac{N^{*}}{2}+1-p_{1}\right)}+\mathcal{O}(\mu), i=1,2 .
$$

Therefore, under conditions (4.1) and (5.1), equation (5.2) holds.

Proof of Theorem 5.2: Similar to that of Theorem 5.1. Equation (5.3) follows directly from (C.2) and (4.4).

\section{APPENDIX D. PROOFS FOR SECTION 7}

Proof of Theorem 7.1: Consider an assembly system (i)-(iv) with (4.1) taking place. Assume that the workforce distribution is $p_{i}^{*}, i=0,1,2$, where $p_{i}^{*}$ are defined by (4.4). Modify the workforce distribution to $p_{i}=$ $g p_{i}^{*}$ and $p_{j}=(1 / g) p_{j}^{*}, i=0,1,2$, and $j=0,1,2$, where $g \neq 0$. Then, we find that the total workforce $p^{*}$ is preserved and is not depending on $g$, but that the line in unimprovable when $g=1$. That is, the production rate achieves its maximum value at $g=1$. Let $P R=P R(g)$. Consequently, we have

$$
\left.\frac{\partial P R(g)}{\partial g}\right|_{g=1}=0 .
$$

By the chain rule,

$$
\begin{aligned}
\left.\frac{\partial P R(g)}{\partial g}\right|_{g=1} & =\left.\left(\frac{\partial P R(g)}{\partial\left(g p_{i}^{*}\right)} \frac{\partial\left(g p_{i}^{*}\right)}{\partial g}+\frac{\partial P R(g)}{\partial p_{j}^{*}} \frac{\left(\frac{p_{j}^{*}}{g}\right)}{\partial g}\right)\right|_{g=1} \\
& =\left.\left(p_{i}^{*} \frac{\partial P R(g)}{\partial p_{i}}-p_{j}^{*}\left(\frac{1}{g}\right)^{2} \frac{\partial P R(g)}{\partial p_{j}}\right)\right|_{g=1}, i=0,1,2, j=0,1,2 .
\end{aligned}
$$


From (D.1),

$$
\left.p_{i}^{*} \frac{\partial P R(g)}{\partial p_{i}}\right|_{g=1}-\left.p_{j}^{*} \frac{\partial P R(g)}{\partial p_{j}}\right|_{g=1}=0
$$

Since $i$ and $j$ are chosen arbitrarily, we therefore conclude that

$$
\left.p_{i}^{*} \frac{\partial P R(g)}{\partial p_{i}}\right|_{g=1}=\left.p_{j}^{*} \frac{\partial P R(g)}{\partial p_{j}}\right|_{g=1}, i=0,1,2, j=0,1,2 .
$$

\section{APPENDIX E. PROOFS FOR SECTION 8}

To prove Theorem 8.1 , we need three auxiliary facts:

Lemma E.1: The following is true:

$$
\begin{aligned}
& \frac{\partial p_{0}\left[1-X_{2}(0)\right]}{\partial p_{1}}=\frac{p_{0}^{2} g_{2}\left(p_{2}, p_{0}\left[1-X_{1}(0)\right], N_{2}\right) g_{1}\left(p_{1}, p_{0}\left[1-X_{2}(0)\right], N_{1}\right)}{1-p_{0}^{2} g_{2}\left(p_{2}, p_{0}\left[1-X_{1}(0)\right], N_{2}\right) g_{2}\left(p_{1}, p_{0}\left[1-X_{2}(0)\right], N_{1}\right)}, \\
& \frac{\partial p_{0}\left[1-X_{1}(0)\right]}{\partial p_{2}}=\frac{p_{0}^{2} g_{2}\left(p_{1}, p_{0}\left[1-X_{2}(0)\right], N_{1}\right) g_{1}\left(p_{2}, p_{0}\left[1-X_{1}(0)\right], N_{2}\right)}{1-p_{0}^{2} g_{2}\left(p_{1}, p_{0}\left[1-X_{2}(0)\right], N_{1}\right) g_{2}\left(p_{2}, p_{0}\left[1-X_{1}(0)\right], N_{2}\right)}, \\
& \frac{\partial p_{0}\left[1-X_{2}(0)\right]}{\partial p_{0}}=\frac{1-Q\left(p_{2}, p_{0}\left[1-X_{1}(0)\right], N_{2}\right)}{1-p_{0}^{2} g_{2}\left(p_{2}, p_{0}\left[1-X_{1}(0)\right], N_{2}\right) g_{2}\left(p_{1}, p_{0}\left[1-X_{2}(0)\right], N_{1}\right)}, \\
& -\frac{\left[1-Q\left(p_{1}, p_{0}\left[1-X_{2}(0)\right], N_{1}\right)\right] p_{0} g_{2}\left(p_{2}, p_{0}\left[1-X_{1}(0)\right], N_{2}\right)}{1-p_{0}^{2} g_{2}\left(p_{2}, p_{0}\left[1-X_{1}(0)\right], N_{2}\right) g_{2}\left(p_{1}, p_{0}\left[1-X_{2}(0)\right], N_{1}\right)},
\end{aligned}
$$

$$
\begin{aligned}
\frac{\partial p_{0}\left[1-X_{1}(0)\right]}{\partial p_{0}} & =\frac{1-Q\left(p_{1}, p_{0}\left[1-X_{2}(0)\right], N_{1}\right)}{1-p_{0}^{2} g_{2}\left(p_{2}, p_{0}\left[1-X_{1}(0)\right], N_{2}\right) g_{2}\left(p_{1}, p_{0}\left[1-X_{2}(0)\right], N_{1}\right)}, \\
& -\frac{\left[1-Q\left(p_{2}, p_{0}\left[1-X_{1}(0)\right], N_{2}\right)\right] p_{0} g_{2}\left(p_{1}, p_{0}\left[1-X_{2}(0)\right], N_{1}\right)}{1-p_{0}^{2} g_{2}\left(p_{2}, p_{0}\left[1-X_{1}(0)\right], N_{2}\right) g_{2}\left(p_{1}, p_{0}\left[1-X_{2}(0)\right], N_{1}\right)},
\end{aligned}
$$


where $X_{1}(0)$ and $X_{2}(0)$ are the steady states of recursive procedure (3.3), $Q(x, y, N)$ is defined in (3.3) and

$$
\begin{aligned}
& g_{1}(x, y, N)=\frac{\partial Q(x, y, N)}{\partial x}= \begin{cases}\frac{-1+\alpha^{N}+N \frac{y-x}{y(1-x)} \alpha^{N}}{y\left(1-\frac{x}{y} \alpha^{N}\right)^{2}}, x \neq y, \\
\frac{-N(N+1)}{2 x(N+1-x)^{2}}, & x=y,\end{cases} \\
& g_{2}(x, y, N)=\frac{\partial Q(x, y, N)}{\partial y}= \begin{cases}\frac{x-\left[x+N \frac{x(y-x)}{y(1-y)}\right] \alpha^{N}}{y^{2}\left(1-\frac{x}{y} \alpha^{N}\right)^{2}}, & x \neq y, \\
\frac{N(N+1-2 x)}{2 x(N+1-x)^{2}}, & x=y,\end{cases}
\end{aligned}
$$

where $\alpha=x(1-y) / y(1-x)$.

Proof: The expression for $\partial p_{0}\left[1-X_{2}(0)\right] / \partial p_{1}$ is derived as follows: From (A.24),

$$
\begin{aligned}
\frac{\partial p_{0}\left[1-X_{2}(0)\right]}{\partial p_{1}} & =\frac{\partial p_{0}\left[1-Q\left(p_{2}, p_{0}\left[1-X_{1}(0)\right], N_{2}\right)\right]}{\partial p_{1}} \\
= & -p_{0} \frac{\partial Q\left(p_{2}, p_{0}\left[1-X_{1}(0)\right], N_{2}\right)}{\partial p_{1}} \\
= & -p_{0}\left[\frac{\partial Q\left(p_{2}, p_{0}\left[1-X_{1}(0)\right], N_{2}\right)}{\partial p_{2}} \frac{\partial p_{2}}{\partial p_{1}}\right] \\
& -p_{0}\left[\frac{\partial Q\left(p_{2}, p_{0}\left[1-X_{1}(0)\right], N_{2}\right)}{\partial p_{0}\left[1-X_{1}(0)\right]} \frac{\partial p_{0}\left[1-X_{1}(0)\right]}{\partial p_{1}}\right] .
\end{aligned}
$$

Since $\partial p_{2} / \partial p_{1}=0$, by Lemma B.1 of [6],

$$
\frac{\partial p_{0}\left[1-X_{2}(0)\right]}{\partial p_{1}}=-p_{0} g_{2}\left(p_{2}, p_{0}\left[1-X_{1}(0)\right], N_{2}\right) \frac{\partial p_{0}\left[1-X_{1}(0)\right]}{\partial p_{1}}
$$


where $g_{2}(x, y, N)$ is given by (E.2). From (A.23),

$$
\begin{aligned}
& \frac{\partial p_{0}\left[1-X_{2}(0)\right]}{\partial p_{1}} \\
& \quad=-p_{0} g_{2}\left(p_{2}, p_{0}\left[1-X_{1}(0)\right], N_{2}\right) \frac{\partial p_{0}\left[1-Q\left(p_{1}, p_{0}\left[1-X_{2}(0)\right], N_{1}\right)\right]}{\partial p_{1}} \\
& \quad=-p_{0} g_{2}\left(p_{2}, p_{0}\left[1-X_{1}(0)\right], N_{2}\right)\left[-p_{0} \frac{d Q\left(p_{1}, p_{0}\left[1-X_{2}(0)\right], N_{1}\right)}{d p_{1}}\right] \\
& \quad=-p_{0} g_{2}\left(p_{2}, p_{0}\left[1-X_{1}(0)\right], N_{2}\right) \\
& \left.\quad-p_{0} \frac{\partial Q\left(p_{1}, p_{0}\left[1-X_{2}(0)\right], N_{1}\right)}{\partial p_{1}} \frac{\partial p_{1}}{\partial p_{1}}-p_{0} \frac{\partial Q\left(p_{1}, p_{0}\left[1-X_{2}(0)\right], N_{1}\right)}{\partial p_{0}\left[1-X_{2}(0)\right]} \frac{\partial p_{0}\left[1-X_{2}(0)\right]}{\partial p_{1}}\right] \\
& \quad=p_{0}^{2} g_{2}\left(p_{2}, p_{0}\left[1-X_{1}(0)\right], N_{2}\right) g_{1}\left(p_{1}, p_{0}\left[1-X_{2}(0)\right], N_{1}\right) \\
& \quad+p_{0}^{2} g_{2}\left(p_{2}, p_{0}\left[1-X_{1}(0)\right], N_{2}\right) g_{2}\left(p_{1}, p_{0}\left[1-X_{2}(0)\right], N_{1}\right) \frac{\partial p_{0}\left[1-X_{2}(0)\right]}{\partial p_{1}} .
\end{aligned}
$$

Therefore, solving (E.3) for $\partial p_{0}\left[1-X_{2}(0)\right] / \partial p_{1}$,

$$
\frac{\partial p_{0}\left[1-X_{2}(0)\right]}{\partial p_{1}}=\frac{p_{0}^{2} g_{2}\left(p_{2}, p_{0}\left[1-X_{1}(0)\right], N_{2}\right) g_{1}\left(p_{1}, p_{0}\left[1-X_{2}(0)\right], N_{1}\right)}{1-p_{0}^{2} g_{2}\left(p_{2}, p_{0}\left[1-X_{1}(0)\right], N_{2}\right) g_{2}\left(p_{1}, p_{0}\left[1-X_{2}(0)\right], N_{1}\right)}
$$

The expression of $\partial p_{0}\left[1-X_{1}(0)\right] / \partial p_{2}$ is obtained analogously.

The expressions for $\partial p_{0}\left[1-X_{2}(0)\right] / \partial p_{0}$ and $\partial p_{0}\left[1-X_{1}(0)\right] / \partial p_{0}$ are derived as follows:

$$
\begin{aligned}
\frac{\partial p_{0}\left[1-X_{2}(0)\right]}{\partial p_{0}} & =\frac{\partial p_{0}\left[1-Q\left(p_{2}, p_{0}\left[1-X_{1}(0)\right], N_{2}\right)\right]}{\partial p_{0}} \\
& =1-Q\left(p_{2}, p_{0}\left[1-X_{1}(0)\right], N_{2}\right) \\
& -p_{0} \frac{\partial Q\left(p_{2}, p_{0}\left[1-X_{1}(0)\right], N_{2}\right)}{\partial p_{0}}
\end{aligned}
$$




$$
\begin{aligned}
& =1-Q\left(p_{2}, p_{0}\left[1-X_{1}(0)\right], N_{2}\right) \\
& -p_{0}\left[\frac{\partial Q\left(p_{2}, p_{0}\left[1-X_{1}(0)\right], N_{2}\right)}{\partial p_{2}} \frac{\partial p_{2}}{\partial p_{0}}\right. \\
& \left.+\frac{\partial Q\left(p_{2}, p_{0}\left[1-X_{1}(0)\right], N_{2}\right)}{\partial p_{0}\left[1-X_{1}(0)\right]} \frac{\partial p_{0}\left[1-X_{1}(0)\right]}{\partial p_{0}}\right], \\
& \frac{\partial p_{0}\left[1-X_{1}(0)\right]}{\partial p_{0}}=\frac{\partial p_{0}\left[1-Q\left(p_{1}, p_{0}\left[1-X_{2}(0)\right], N_{1}\right)\right]}{\partial p_{0}} \\
& =1-Q\left(p_{1}, p_{0}\left[1-X_{2}(0)\right], N_{1}\right) \\
& -p_{0} \frac{\partial Q\left(p_{1}, p_{0}\left[1-X_{2}(0)\right], N_{1}\right]}{\partial p_{0}} \\
& =1-Q\left(p_{1}, p_{0}\left[1-X_{2}(0)\right], N_{1}\right) \\
& -p_{0}\left[\frac{\partial Q\left(p_{1}, p_{0}\left[1-X_{2}(0)\right], N_{1}\right)}{\partial p_{1}} \frac{\partial p_{1}}{\partial p_{0}}\right. \\
& \left.+\frac{\partial Q\left(p_{1}, p_{0}\left[1-X_{2}(0)\right], N_{1}\right)}{\partial p_{0}\left[1-X_{2}(0)\right]} \frac{\partial p_{0}\left[1-X_{2}(0)\right]}{\partial p_{0}}\right] \text {. }
\end{aligned}
$$

Since $\partial p_{2} / \partial p_{0}=0$ and $\partial p_{1} / \partial p_{0}=0$, using Lemma B.1 of [1],

$$
\begin{aligned}
\frac{\partial p_{0}\left[1-X_{2}(0)\right]}{\partial p_{0}} & =1-Q\left(p_{2}, p_{0}\left[1-X_{1}(0)\right], N_{2}\right) \\
& -p_{0} g_{2}\left(p_{2}, p_{0}\left[1-X_{1}(0)\right], N_{2}\right) \frac{\partial p_{0}\left[1-X_{1}(0)\right]}{\partial p_{0}}
\end{aligned}
$$

$$
\begin{aligned}
\frac{\partial p_{0}\left[1-X_{1}(0)\right]}{\partial p_{0}} & =1-Q\left(p_{1}, p_{0}\left[1-X_{2}(0)\right], N_{1}\right) \\
& -p_{0} g_{2}\left(p_{1}, p_{0}\left[1-X_{2}(0)\right], N_{1}\right) \frac{\partial p_{0}\left[1-X_{2}(0)\right]}{\partial p_{0}}
\end{aligned}
$$


where $g_{2}(x, y, N)$ is defined by (E.2). Equations (E.4) and (E.5) can be re-written in the matrix form as follows:

$$
\begin{gathered}
{\left[p_{0} g_{2}\left(p_{1}, p_{0}\left[1-X_{2}(0)\right], N_{1}\right)^{p_{0} g_{2}\left(p_{2}, p_{0}\left[1-X_{1}(0)\right], N_{2}\right)} \begin{array}{c}
1
\end{array}\right]\left[\begin{array}{c}
\frac{\partial p_{0}\left[1-X_{2}(0)\right]}{\partial p_{0}} \\
\left.\frac{\partial p_{0}\left[1-X_{1}(0)\right]}{\partial p_{0}}\right]
\end{array}\right.} \\
=\left[\begin{array}{c}
1-Q\left(p_{2}, p_{0}\left[1-X_{1}(0)\right], N_{2}\right) \\
1-Q\left(p_{1}, p_{0}\left[1-X_{2}(0)\right], N_{1}\right)
\end{array}\right] .
\end{gathered}
$$

Solving (E.6) for $\partial p_{0}\left[1-X_{2}(0)\right] / \partial p_{0}$ and $\partial p_{0}\left[1-X_{1}(0)\right] / \partial p_{0}$, we obtain

$$
\begin{gathered}
\frac{\partial p_{0}\left[1-X_{2}(0)\right]}{\partial p_{0}}=\frac{1-Q\left(p_{2}, p_{0}\left[1-X_{1}(0)\right], N_{2}\right)}{1-p_{0}^{2} g_{2}\left(p_{2}, p_{0}\left[1-X_{1}(0)\right], N_{2}\right) g_{2}\left(p_{1}, p_{0}\left[1-X_{2}(0)\right], N_{1}\right)} \\
-\frac{\left[1-Q\left(p_{1}, p_{0}\left[1-X_{2}(0)\right], N_{1}\right)\right] p_{0} g_{2}\left(p_{2}, p_{0}\left[1-X_{1}(0)\right], N_{2}\right)}{1-p_{0}^{2} g_{2}\left(p_{2}, p_{0}\left[1-X_{1}(0)\right], N_{2}\right) g_{2}\left(p_{1}, p_{0}\left[1-X_{2}(0)\right], N_{1}\right)}, \\
\frac{\partial p_{0}\left[1-X_{1}(0)\right]}{\partial p_{0}}=\frac{1-Q\left(p_{1}, p_{0}\left[1-X_{2}(0)\right], N_{1}\right)}{1-p_{0}^{2} g_{2}\left(p_{2}, p_{0}\left[1-X_{1}(0)\right], N_{2}\right) g_{2}\left(p_{1}, p_{0}\left[1-X_{2}(0)\right], N_{1}\right)} \\
-\frac{\left[1-Q\left(p_{2}, p_{0}\left[1-X_{1}(0)\right], N_{2}\right)\right] p_{0} g_{2}\left(p_{1}, p_{0}\left[1-X_{2}(0)\right], N_{1}\right)}{1-p_{0}^{2} g_{2}\left(p_{2}, p_{0}\left[1-X_{1}(0)\right], N_{2}\right) g_{2}\left(p_{1}, p_{0}\left[1-X_{2}(0)\right], N_{1}\right)} .
\end{gathered}
$$

Lemma E.2: For assembly system (i)-(vi),

$$
\begin{aligned}
& \frac{\partial P R}{\partial p_{1}}=1-Q\left(p_{0}\left[1-X_{2}(0)\right], p_{1}, N_{1}\right) \\
& -p_{1}\left[g_{1}\left(p_{0}\left[1-X_{2}(0)\right], p_{1}, N_{1}\right) \frac{\partial p_{0}\left[1-X_{2}(0)\right]}{\partial p_{1}}+g_{2}\left(p_{0}\left[1-X_{2}(0)\right], p_{1}, N_{1}\right)\right],
\end{aligned}
$$




$$
\begin{aligned}
& \frac{\partial P R}{\partial p_{2}}=1-Q\left(p_{0}\left[1-X_{1}(0)\right], p_{2}, N_{2}\right) \\
& -p_{2}\left[g_{1}\left(p_{0}\left[1-X_{1}(0)\right], p_{2}, N_{2}\right) \frac{\partial p_{0}\left[1-X_{1}(0)\right]}{\partial p_{2}}+g_{2}\left(p_{0}\left[1-X_{1}(0)\right], p_{2}, N_{2}\right)\right], \\
& \quad \frac{\partial P R}{\partial p_{0}}=\left[1-Q\left(p_{1}, p_{0}\left[1-X_{2}(0)\right], N_{1}\right)\right]\left[1-Q\left(p_{2}, p_{0}\left[1-X_{1}(0)\right], N_{2}\right)\right] \\
& -p_{0}\left[1-Q\left(p_{2}, p_{0}\left[1-X_{1}(0)\right], N_{2}\right)\right] g_{2}\left(p_{1}, p_{0}\left[1-X_{2}(0)\right], N_{1}\right) \frac{\partial p_{0}\left[1-X_{2}(0)\right]}{\partial p_{0}} \\
& -p_{0}\left[1-Q\left(p_{1}, p_{0}\left[1-X_{2}(0)\right], N_{1}\right)\right] g_{2}\left(p_{2}, p_{0}\left[1-X_{1}(0)\right], N_{2}\right) \frac{\partial p_{0}\left[1-X_{1}(0)\right]}{\partial p_{0}},
\end{aligned}
$$

where $\partial p_{0}\left[1-X_{2}(0)\right] / \partial p_{1}, \partial p_{0}\left[1-X_{1}(0)\right] / \partial p_{2}, \partial p_{0}\left[1-X_{2}(0)\right] / \partial p_{0}$, $\partial p_{0}\left[1-X_{1}(0)\right] / \partial p_{0}, g_{1}(x, y, N)$ and $g_{2}(x, y, N)$ are given by $(E .1)$ and (E.2), respectively.

Proof: By Lemma A. 4,

$$
\begin{aligned}
\frac{\partial P R}{\partial p_{1}}= & \frac{\partial p_{1}\left[1-Q\left(p_{0}\left[1-X_{2}(0)\right], p_{1}, N_{1}\right)\right]}{\partial p_{1}} \\
= & 1-Q\left(p_{0}\left[1-X_{2}(0)\right], p_{1}, N_{1}\right)-p_{1} \frac{d Q\left(p_{0}\left[1-X_{2}(0)\right], p_{1}, N_{1}\right)}{d p_{1}} \\
= & 1-Q\left(p_{0}\left[1-X_{2}(0)\right], p_{1}, N_{1}\right) \\
-p_{1}\left[\frac{\partial Q\left(p_{0}\left[1-X_{2}(0)\right], p_{1}, N_{1}\right)}{\partial p_{0}\left[1-X_{2}(0)\right]} \frac{\partial p_{0}\left[1-X_{2}(0)\right]}{\partial p_{1}}\right. & \\
& \left.+\frac{\partial Q\left(p_{0}\left[1-X_{2}(0)\right], p_{1}, N_{1}\right)}{\partial p_{1}}\right],
\end{aligned}
$$




$$
\begin{aligned}
\frac{\partial P R}{\partial p_{2}} & =\frac{\partial p_{2}\left[1-Q\left(p_{0}\left[1-X_{1}(0)\right], p_{2}, N_{2}\right)\right]}{\partial p_{2}} \\
& =1-Q\left(p_{0}\left[1-X_{1}(0)\right], p_{2}, N_{2}\right)-p_{2} \frac{d Q\left(p_{0}\left[1-X_{1}(0)\right], p_{2}, N_{2}\right)}{d p_{2}} \\
& =1-Q\left(p_{0}\left[1-X_{1}(0)\right], p_{2}, N_{2}\right) \\
& -p_{2}\left[\frac{\partial Q\left(p_{0}\left[1-X_{1}(0)\right], p_{2}, N_{2}\right)}{\partial p_{0}\left[1-X_{1}(0)\right]} \frac{\partial p_{0}\left[1-X_{1}(0)\right]}{\partial p_{2}}\right. \\
& \left.+\frac{\partial Q\left(p_{0}\left[1-X_{1}(0)\right], p_{2}, N_{2}\right)}{\partial p_{2}}\right]
\end{aligned}
$$

$$
\left.\begin{array}{rl}
\frac{\partial P R}{\partial p_{0}}= & \frac{\partial p_{0}\left[1-Q\left(p_{1}, p_{0}\left[1-X_{2}(0)\right], N_{1}\right)\right]\left[1-Q\left(p_{2}, p_{0}\left[1-X_{1}(0)\right], N_{2}\right)\right]}{\partial p_{0}} \\
= & {\left[1-Q\left(p_{1}, p_{0}\left[1-X_{2}(0)\right], N_{1}\right)\right]\left[1-Q\left(p_{2}, p_{0}\left[1-X_{1}(0)\right], N_{2}\right)\right]} \\
& -p_{0}\left[1-Q\left(p_{2}, p_{0}\left[1-X_{1}(0)\right], N_{2}\right)\right] \frac{\partial Q\left(p_{1}, p_{0}\left[1-X_{2}(0)\right], N_{1}\right)}{\partial p_{0}} \\
& -p_{0}\left[1-Q\left(p_{1}, p_{0}\left[1-X_{2}(0)\right], N_{1}\right)\right] \frac{\partial Q\left(p_{2}, p_{0}\left[1-X_{1}(0)\right], N_{2}\right)}{\partial p_{0}} \\
= & -p_{0}\left[1-Q\left(p_{1}, p_{0}\left[1-x_{0}\left[1-X_{1}(0)\right], N_{2}\right)\right]\right. \\
& \quad\left[\frac{\partial Q\left(p_{1}, p_{0}\left[1-X_{2}(0)\right], N_{1}\right)}{\partial p_{1}} \frac{\partial p_{1}}{\partial p_{0}}\right. \\
& \left.\quad \frac{\partial Q\left(p_{1}, p_{0}\left[1-X_{2}(0)\right], N_{1}\right)}{\partial p_{0}\left[1-X_{2}(0)\right]} \frac{\partial p_{0}\left[1-X_{2}(0)\right]}{\partial p_{0}}\right] \\
& -p_{0}\left[1-Q\left(p_{1}, p_{0}\left[1-X_{2}(0)\right], N_{1}\right)\right]
\end{array}\right]
$$




$$
\begin{aligned}
& \left.+\frac{\partial Q\left(p_{2}, p_{0}\left[1-X_{1}(0)\right], N_{2}\right)}{\partial p_{0}\left[1-X_{1}(0)\right]} \frac{\partial p_{0}\left[1-X_{1}(0)\right]}{\partial p_{0}}\right] \\
& =\left[1-Q\left(p_{1}, p_{0}\left[1-X_{2}(0)\right], N_{1}\right)\right]\left[1-Q\left(p_{2}, p_{0}\left[1-X_{1}(0)\right], N_{2}\right)\right] \\
& -p_{0}\left[1-Q\left(p_{2}, p_{0}\left[1-X_{1}(0)\right], N_{2}\right)\right] \\
& \frac{\partial Q\left(p_{1}, p_{0}\left[1-X_{2}(0)\right], N_{1}\right)}{\partial p_{0}\left[1-X_{2}(0)\right]} \frac{\partial p_{0}\left[1-X_{2}(0)\right]}{\partial p_{0}} \\
& -p_{0}\left[1-Q\left(p_{1}, p_{0}\left[1-X_{2}(0)\right], N_{1}\right)\right] \\
& \frac{\partial Q\left(p_{2}, p_{0}\left[1-X_{1}(0)\right], N_{2}\right)}{\partial p_{0}\left[1-X_{1}(0)\right]} \frac{\partial p_{0}\left[1-X_{1}(0)\right]}{\partial p_{0}} \text {. }
\end{aligned}
$$

Using the expressions for $\partial Q(x, y, N) / \partial x$ and $\partial Q(x, y, N) / \partial y$ from (E.2), and the expressions for $\partial p_{0}\left[1-X_{2}(0)\right] / \partial p_{1}, \partial p_{0}\left[1-X_{1}(0)\right] / \partial p_{2}, \partial p_{0}[1-$ $\left.X_{2}(0)\right] / \partial p_{0}$, and $\partial p_{0}\left[1-X_{1}(0)\right] / \partial p_{0}$ from (E.1), (E.8) is reduced to (E.7).

LEMMA E.3: Under Hypothesis 8.1, the following hold:

(a) if $m b_{j}>m s_{j}$, then

$$
g_{2}\left(p_{j}, p_{0}\left[1-X_{3-j}(0)\right], N_{j}\right) \frac{\partial p_{0}\left[1-X_{3-j}(0)\right]}{\partial p_{0}} \sim \mathcal{O}(\epsilon), j=1,2
$$

(b) if $m b_{j}<m s_{j}$, then

$$
\begin{gathered}
g_{1}\left(p_{0}\left[1-X_{3-j}(0)\right], p_{j}, N_{j}\right) \frac{\partial p_{0}\left[1-X_{3-j}(0)\right]}{\partial p_{j}}+g_{2}\left(p_{0}\left[1-X_{3-j}(0)\right], p_{j}, N_{j}\right) \sim \mathcal{O}(\epsilon), \\
j=1,2,
\end{gathered}
$$

where $\epsilon=\max _{j, s}\left(\epsilon_{j 1}, \epsilon_{s 2}\right)$ and $g_{1}(x, y, N), g_{2}(x, y, N), \partial p_{0}\left[1-X_{3-j}(0)\right] / \partial p_{0}$, and $\partial p_{0}\left[1-X_{3-j}(0)\right] / \partial p_{j}, j=1,2$, are given by $(E .2)$ and $(E .1)$, respectively.

Proof: Statement (a) is proved as follows: Under Hypothesis 8.1, 


$$
\begin{aligned}
\epsilon_{j 1}= & Q\left(p_{j}, p_{0}\left[1-X_{3-j}(0)\right], N_{j}\right)=\frac{\left(1-p_{j}\right)(1-\alpha)}{1-\frac{p_{j}}{p_{0}\left[1-X_{3-j}(0)\right]} \alpha^{N_{j}}} \\
= & \frac{\frac{p_{0}\left[1-X_{3-j}(0)\right]-p_{j}}{p_{0}\left[1-X_{3-j}(0)\right]}}{1-\frac{p_{j}}{p_{0}\left[1-X_{3-j}(0)\right]} \alpha^{N_{j}}}<<1, j=1,2,
\end{aligned}
$$

where

$$
\alpha=\frac{p_{j}\left(1-p_{0}\left[1-X_{3-j}(0)\right]\right)}{p_{0}\left[1-X_{3-j}(0)\right]\left(1-p_{j}\right)}
$$

Hence,

$$
1-\frac{p_{j}}{p_{0}\left[1-X_{3-j}(0)\right]} \alpha^{N_{j}}=\frac{p_{0}\left[1-X_{3-j}(0)\right]-p_{j}}{\epsilon_{j 1} p_{0}\left[1-X_{3-j}(0)\right]}, j=1,2 .
$$

Using (E.2) and (E.11), we write:

$g_{2}\left(p_{j}, p_{0}\left[1-X_{3-j}(0)\right], N_{j}\right)=$

$$
\frac{\epsilon_{j 1}^{2}\left\{p_{j}-\left[p_{j}+\frac{N_{j}\left(p_{0}\left[1-X_{3-j}(0)\right]-p_{j}\right)}{p_{0}\left[1-X_{3-j}(0)\right]\left(1-p_{0}\left[1-X_{3-j}(0)\right]\right)}\right]\left(1-\frac{p_{0}\left[1-X_{3-j}(0)\right]-p_{j}}{\epsilon_{j 1} p_{0}\left[1-X_{3-j}(0)\right]}\right) \frac{p_{0}\left[1-X_{3-j}(0)\right]}{p_{j}}\right\}}{\left(p_{0}\left[1-X_{3-j}(0)\right]-p_{j}\right)^{2}} \sim \mathcal{O}(\epsilon) .
$$

By Lemma E.1 and (E.12),

$$
\begin{aligned}
& \frac{\partial p_{0}\left[1-X_{3-j}(0)\right]}{\partial p_{0}} \\
& \approx \frac{1-Q\left(p_{3-j}, p_{0}\left[1-X_{j}(0)\right], N_{3-j}\right)-\left(1-\epsilon_{j 1}\right) p_{0} g_{2}\left(p_{3-j}, p_{0}\left[1-X_{j}(0)\right], N_{3-j}\right)}{1-p_{0}^{2} g_{2}\left(p_{3-j}, p_{0}\left[1-X_{j}(0)\right], N_{3-j}\right) \mathcal{O}(\epsilon)}
\end{aligned}
$$

Neglecting the $\mathcal{O}(\boldsymbol{\epsilon})$ terms, we have 


$$
\begin{gathered}
\frac{\partial p_{0}\left[1-X_{3-j}(0)\right]}{\partial p_{0}} \approx 1-Q\left(p_{3-j}, p_{0}\left[1-X_{j}(0)\right], N_{3-j}\right) \\
-p_{0} g_{2}\left(p_{3-j}, p_{0}\left[1-X_{j}(0)\right], N_{3-j}\right) .
\end{gathered}
$$

Therefore, substituting (E.13) into the left hand side of (E.9) and using (E.12), we conclude that

$$
g_{2}\left(p_{j}, p_{0}\left[1-X_{3-j}(0)\right], N_{j}\right) \frac{\partial p_{0}\left[1-X_{3-j}(0)\right]}{\partial p_{0}} \sim \mathcal{O}(\epsilon), j=1,2
$$

Statement (b) is proved similarly.

Proof of Theorem 8.1: Under the assumption that system (i)-(vi) has a unique machine, $m_{i}, i \mathrm{E}\{0,1,2\}$, with no arrows emanating from it, the following three cases are possible:

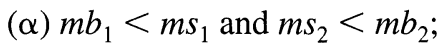

$$
\begin{aligned}
& \text { (ß) } m b_{1}>m s_{1} \text { and } m s_{2}<m b_{2} \text {; } \\
& (\gamma) m b_{1}>m s_{1} \text { and } m s_{2}>m b_{2} \text {. }
\end{aligned}
$$

Consider first case $(\alpha)$. Under Hypothesis 8.1, using Lemmas E.2 and E.3, for the first component machine,

$$
\begin{aligned}
\frac{\partial P R}{\partial p_{1}}=1 & -Q\left(p_{0}\left[1-X_{2}(0)\right], p_{1}, N_{1}\right) \\
& -p_{1}\left[g_{1}\left(p_{0}\left[1-X_{2}(0)\right], p_{1}, N_{1}\right) \frac{\partial p_{0}\left[1-X_{2}(0)\right]}{\partial p_{1}}\right. \\
& \left.+g_{2}\left(p_{0}\left[1-X_{2}(0)\right], p_{1}, N_{1}\right)\right] \\
=1-\epsilon_{12}- & p_{1}\left[g_{1}\left(p_{0}\left[1-X_{2}(0)\right], p_{1}, N_{1}\right) \frac{\partial p_{0}\left[1-X_{2}(0)\right]}{\partial p_{1}}\right.
\end{aligned}
$$




$$
\left.+g_{2}\left(p_{0}\left[1-X_{2}(0)\right], p_{1}, N_{1}\right)\right]
$$

$$
=1-\alpha_{1},
$$

where

$$
\begin{aligned}
\alpha_{1}=\epsilon_{12} & +p_{1}\left[g_{1}\left(p_{0}\left[1-X_{2}(0)\right], p_{1}, N_{1}\right) \frac{\partial p_{0}\left[1-X_{2}(0)\right]}{\partial p_{1}}\right. \\
& \left.+g_{2}\left(p_{0}\left[1-X_{2}(0)\right], p_{1}, N_{1}\right)\right] \sim \mathcal{O}(\epsilon) .
\end{aligned}
$$

Analogously, for the second component machine,

$$
\begin{aligned}
& \frac{\partial P R}{\partial p_{2}}=1-Q\left(p_{0}\left[1-X_{1}(0)\right], p_{2}, N_{2}\right) \\
& -p_{2}\left[g_{1}\left(p_{0}\left[1-X_{1}(0)\right], p_{2}, N_{2}\right) \frac{\partial p_{0}\left[1-X_{1}(0)\right]}{\partial p_{2}}+g_{2}\left(p_{0}\left[1-X_{1}(0)\right], p_{2}, N_{2}\right)\right] \\
& \quad=1-\alpha_{2},
\end{aligned}
$$

where

$$
\begin{aligned}
& \alpha_{2}=Q\left(p_{0}\left[1-X_{1}(0)\right], p_{2}, N_{2}\right) \\
& \begin{array}{c}
+p_{2}\left[g_{1}\left(p_{0}\left[1-X_{1}(0)\right], p_{2}, N_{2}\right) \frac{\partial p_{0}\left[1-X_{1}(0)\right]}{\partial p_{2}}+g_{2}\left(p_{0}\left[1-X_{1}(0)\right], p_{2}, N_{2}\right)\right] \\
\sim \mathcal{O}(1) .
\end{array}
\end{aligned}
$$

Finally, for the assembly machine,

$$
\begin{aligned}
& \frac{\partial P R}{\partial p_{0}}=\left[1-Q\left(p_{1}, p_{0}\left[1-X_{2}(0)\right], N_{1}\right)\right]\left[1-Q\left(p_{2}, p_{0}\left[1-X_{1}(0)\right], N_{2}\right)\right] \\
& -p_{0}\left[1-Q\left(p_{2}, p_{0}\left[1-X_{1}(0)\right], N_{2}\right)\right] g_{2}\left(p_{1}, p_{0}\left[1-X_{2}(0)\right], N_{1}\right) \frac{\partial p_{0}\left[1-X_{2}(0)\right]}{\partial p_{0}} \\
& -p_{0}\left[1-Q\left(p_{1}, p_{0}\left[1-X_{2}(0)\right], N_{1}\right)\right]
\end{aligned}
$$




$$
\begin{aligned}
& g_{2}\left(p_{2}, p_{0}\left[1-X_{1}(0)\right], N_{2}\right) \frac{\partial p_{0}\left[1-X_{1}(0)\right]}{\partial p_{0}} \\
& \quad=\left[1-Q\left(p_{1}, p_{0}\left[1-X_{2}(0)\right], N_{1}\right)\right]\left(1-\epsilon_{21}\right) \\
& \quad-p_{0}\left(1-\epsilon_{21}\right) g_{2}\left(p_{1}, p_{0}\left[1-X_{2}(0)\right], N_{1}\right) \frac{\partial p_{0}\left[1-X_{2}(0)\right]}{\partial p_{0}} \\
& \quad-p_{0}\left[1-Q\left(p_{1}, p_{0}\left[1-X_{2}(0)\right], N_{1}\right)\right] \\
& g_{2}\left(p_{2}, p_{0}\left[1-X_{1}(0)\right], N_{2}\right) \frac{\partial p_{0}\left[1-X_{1}(0)\right]}{\partial p_{0}} \\
& \quad=1-\alpha_{0},
\end{aligned}
$$

where

$$
\begin{aligned}
& \alpha_{0}= Q\left(p_{1}, p_{0}\left[1-X_{2}(0)\right], N_{1}\right)\left(1-\epsilon_{21}\right) \\
&+p_{0}\left(1-\epsilon_{21}\right) g_{2}\left(p_{1}, p_{0}\left[1-X_{2}(0)\right], N_{1}\right) \frac{\partial p_{0}\left[1-X_{2}(0)\right]}{\partial p_{0}} \\
&+p_{0}\left[1-Q\left(p_{1}, p_{0}\left[1-X_{2}(0)\right], N_{1}\right)\right] \\
& g_{2}\left(p_{2}, p_{0}\left[1-X_{1}(0)\right], N_{2}\right) \frac{\partial p_{0}\left[1-X_{1}(0)\right]}{\partial p_{0}} \sim O(1) .
\end{aligned}
$$

Therefore,

$$
\frac{\partial P R}{\partial p_{1}}>\frac{\partial P R}{\partial p_{2}} \text { and } \frac{\partial \mathrm{PR}}{\partial p_{1}}>\frac{\partial \mathrm{PR}}{\partial p_{0}},
$$

i.e., the first component machine is the bottleneck.

Consider now case $(\beta)$. Using Hypothesis 8.1 and Lemmas E.2 and E.3, $\partial P R / \partial \mathrm{p}_{1}$ and $\partial P R / \partial p_{0}$ are estimated as follows: 


$$
\begin{aligned}
\frac{\partial P R}{\partial p_{1}} & =1-Q\left(p_{0}\left[1-X_{2}(0)\right], p_{1}, N_{1}\right) \\
-p_{1} & {\left[g_{1}\left(p_{0}\left[1-X_{2}(0)\right], p_{1}, N_{1}\right) \frac{\partial p_{0}\left[1-X_{2}(0)\right]}{\partial p_{1}}+g_{2}\left(p_{0}\left[1-X_{2}(0)\right], p_{1}, N_{1}\right)\right] } \\
& =1-\alpha_{1},
\end{aligned}
$$

where

$$
\begin{aligned}
\alpha_{1}= & Q\left(p_{0}\left[1-X_{2}(0)\right], p_{1}, N_{1}\right) \\
+ & p_{1}\left[g_{1}\left(p_{0}\left[1-X_{2}(0)\right], p_{1}, N_{1}\right) \frac{\partial p_{0}\left[1-X_{2}(0)\right]}{\partial p_{1}}\right. \\
& \left.\quad+g_{2}\left(p_{0}\left[1-X_{2}(0)\right], p_{1}, N_{1}\right)\right] \sim \mathcal{O}(1) .
\end{aligned}
$$

and

$$
\begin{aligned}
& \frac{\partial P R}{\partial p_{0}}=\left(1-\epsilon_{11}\right)\left(1-\epsilon_{21}\right) \\
& -p_{0}\left(1-\epsilon_{21}\right) g_{2}\left(p_{1}, p_{0}\left[1-X_{2}(0)\right], N_{1}\right) \frac{\partial p_{0}\left[1-X_{2}(0)\right]}{\partial p_{0}} \\
& -p_{0}\left(1-\epsilon_{11}\right) g_{2}\left(p_{2}, p_{0}\left[1-X_{1}(0)\right], N_{2}\right) \frac{\partial p_{0}\left[1-X_{1}(0)\right]}{\partial p_{0}}, \\
& =1-\alpha_{0},
\end{aligned}
$$

where

$$
\begin{aligned}
\alpha_{0}= & \epsilon_{11}+\epsilon_{21}\left(1-\epsilon_{11}\right) \\
& +p_{0}\left(1-\epsilon_{21}\right) g_{2}\left(p_{1}, p_{0}\left[1-X_{2}(0)\right], N_{1}\right) \frac{\partial p_{0}\left[1-X_{2}(0)\right]}{\partial p_{0}} \\
& +p_{0}\left(1-\epsilon_{11}\right) g_{2}\left(p_{2}, p_{0}\left[1-X_{1}(0)\right], N_{2}\right) \frac{\partial p_{0}\left[1-X_{1}(0)\right]}{\partial p_{0}} \sim \mathcal{O}(\epsilon) .
\end{aligned}
$$


The expression for $\partial P R / \partial p_{2}$ remains the same as in case $(\alpha)$, i.e., (E.15). Therefore,

$$
\frac{\partial P R}{\partial p_{0}}>\frac{\partial P R}{\partial p_{1}} \text { and } \frac{\partial P R}{\partial p_{0}}>\frac{\partial P R}{\partial p_{2}}
$$

i.e., the assembly machine is the bottleneck.

Case $(\gamma)$ is similar to case $(\alpha)$ and is proved analogously.

\section{References}

[1] D. Jacobs and S. M. Meerkov, "A System-Theoretic Property of Serial Production Lines: Improvability," International Journal of System Science, Vol. 26, No. 4, pp. 755-785, 1995.

[2] D. Jacobs and S. M. Meerkov, "Mathematical Theory of Improvability for Production Systems," Mathematical Problems in Engineering, Vol. 1, No. 2, pp. 95-137, 1995.

[3] J.-T. Lim and S. M. Meerkov, "On Asymptotically Reliable Closed Serial Production Lines," Control Engineering Practice, Vol. 1, No. 1, pp. 147-152, 1993.

[4] S. M. Meerkov and E. Park, "Improvability Properties of Closed Transfer Lines," Proceeding of the 33rd IEEE CDC, Orlando, FL, Dec. 1994; also Control Group Report No. CGR-94-09, The University of Michigan, Ann Arbor, MI, April 1994.

[5] C.-T. Kuo, J.-T. Lim and S. M. Meerkov, "Improvability Analysis of a Machining Transfer Line: An Application," Proceeding of the 33rd IEEE CDC, Orlando, FL, Dec. 1994; also Control Group Report No. CGR-94-08, The University of Michigan, Ann Arbor, MI, April 1994.

[6] C.-T. Kuo, J.-T. Lim and S. M. Meerkov, "Bottlenecks in Serial Production Lines: A System-Theoretic Approach," Mathematical Problems in Engineering, Vol. 2, No. 3, April 1996.

[7] J. M. Harrison, “Assembly-Like Queues," Journal of Applied Probability, Vol. 10, pp. 354-367, 1973.

[8] K. R. Baker, S. G. Powell and D. F. Pyke, "Buffered and Unbuffered Assembly Systems with Variable Processing Times," J. Manufacturing and Oper. Management, Vol. 3, pp. 200-223, 1990.

[9] M. B. M. de Koster, "Approximation of Assembly-Disassembly Systems," Report BDK/ORS/87-02 of the Department of Industrial Engineering, Eindhoven University of Technology, Netherlands, 1987.

[10] S. B. Gershwin, "Assembly/Disassembly Systems: An Efficient Decomposition Algorithm for Tree-Structured Networks," IIE Transactions, Vol. 23, No. 4, pp. 302-314, December 1991.

[11] M. Di Mascolo, R. David and Y. Dallery, "Modeling and Analysis of Assembly Systems with Unreliable Machines and Finite Buffers," IIE Transactions, Vol. 23, No. 4, pp. 315-331, December 1991.

[12] K. R. Baker, S. G. Powell and D. F. Pyke, "Optimal Allocation of Work in Assembly Systems," Management Science, Vol. 39, pp. 101-106, 1993.

[13] S. G. Powell and D. F. Pyke, "Buffering Unbalanced Assembly Systems," Working Paper No. 308, The Amos Tuck School of Business Administration, Dartmouth College, Hanover, NH, Feb. 1995. 
[14] J. G. Shanthikumar and D. D. Yao, "Monotonicity and Concavity Properties in Cyclic Queueing Networks with Finite Buffers," Queueing Networks with Blocking, eds. H. G. Perros and T. Altiok (North-Holland, Amsterdam), pp. 325-345, 1989.

[15] P. Glasserman and D. Yao, "Algebraic Structure of some Stochastic Discrete Event Systems, with Applications," J. Discrete Dynamic Systems: Theory and Appl., Vol. 1, pp. 7-36, 1991.

[16] R. Bellman, Introduction to Matrix Analysis, McGraw-Hill, NY, 1960. 


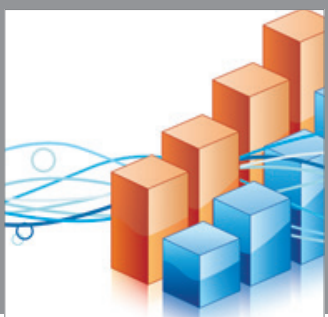

Advances in

Operations Research

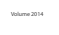

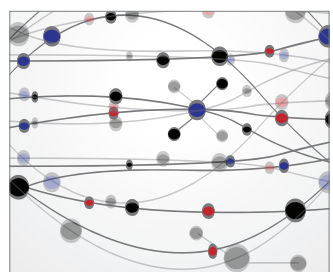

\section{The Scientific} World Journal
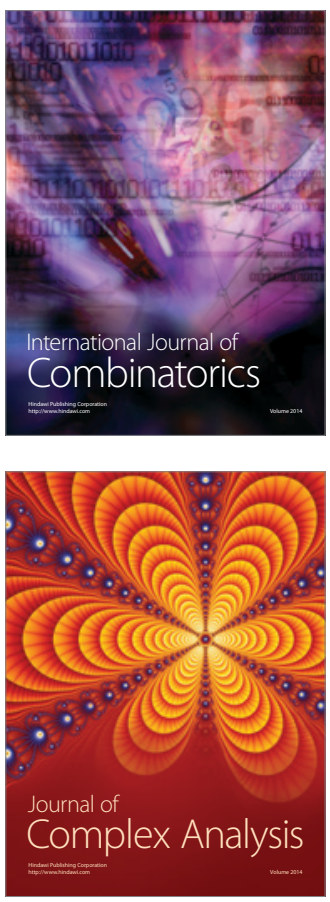

International Journal of

Mathematics and

Mathematical

Sciences
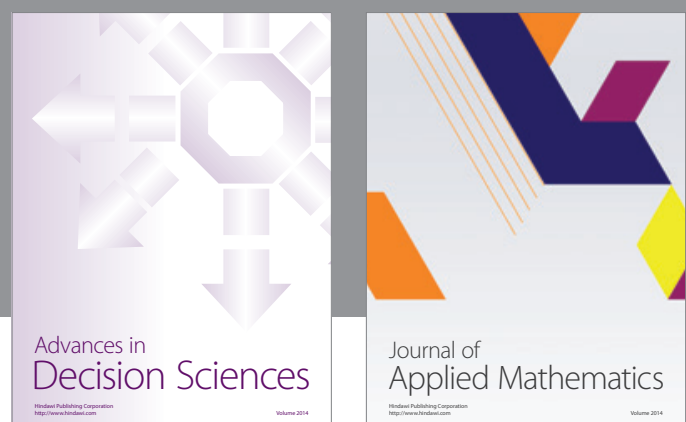

Journal of

Applied Mathematics
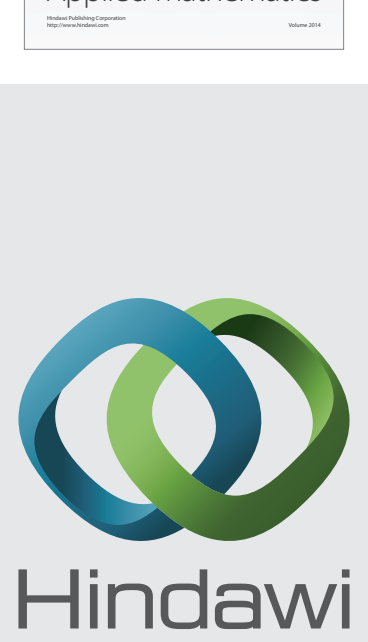

Submit your manuscripts at http://www.hindawi.com
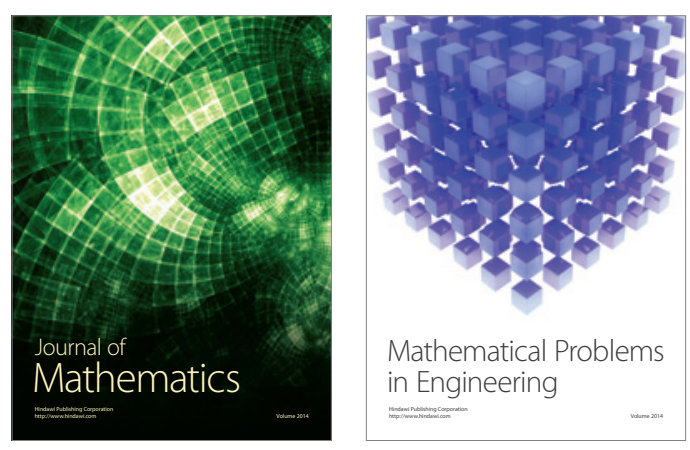

Mathematical Problems in Engineering
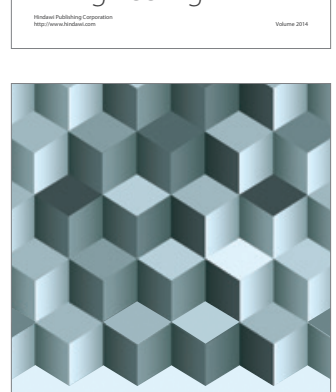

Journal of

Function Spaces
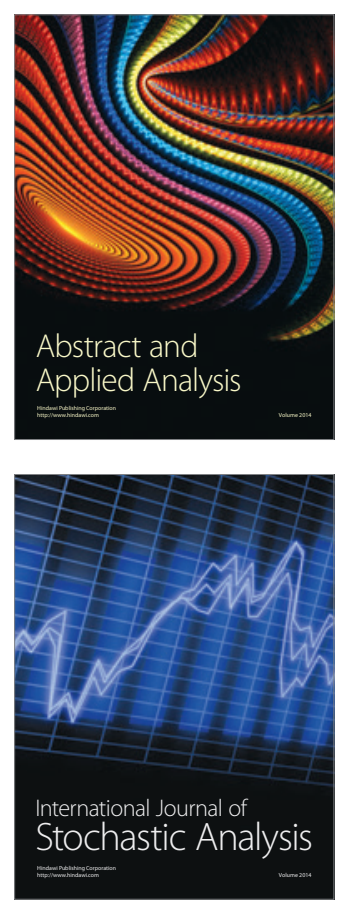

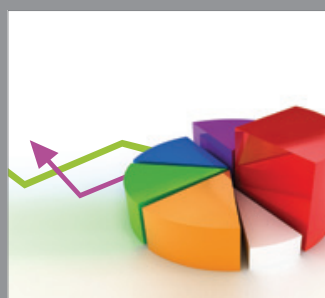

ournal of

Probability and Statistics

Promensencen
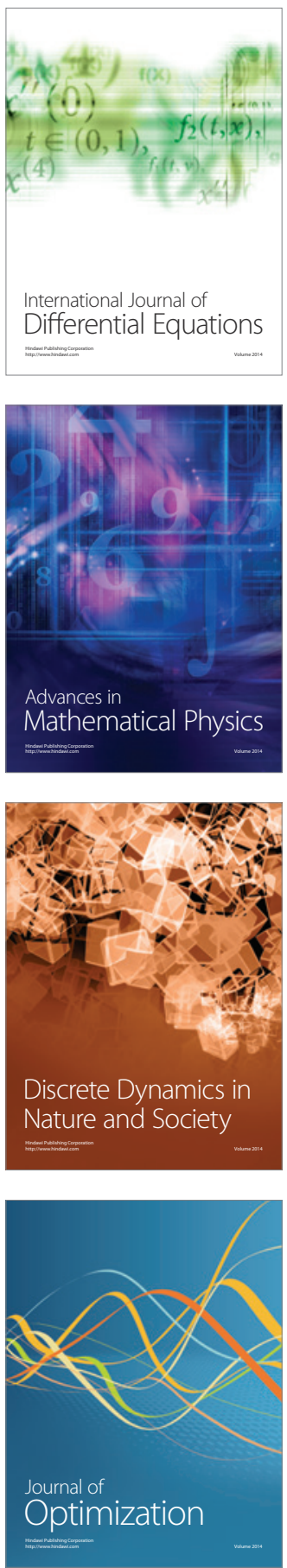